Published in final edited form as:

Nat Protoc. 2019 November ; 14(11): 3033-3058. doi:10.1038/s41596-019-0203-1.

\title{
V2a Interneuron Differentiation from Mouse and Human Pluripotent Stem Cells
}

\author{
Jessica C Butts ${ }^{1,2}$, Nisha lyer ${ }^{3}$, Nick White $^{4}$, Russell Thompson ${ }^{5}$, Shelly Sakiyama-Elbert ${ }^{6}$, \\ Todd C McDevitt ${ }^{7,8}$ \\ ${ }^{1}$ Gladstone Institutes, San Francisco, CA, USA. \\ ${ }^{2}$ Graduate Program in Bioengineering, University of California, San Francisco and University of \\ California, Berkeley, CA, USA. \\ ${ }^{3}$ Department of Biomedical Engineering, University of Wisconsin-Madison, Madison, WI, USA. \\ ${ }^{4}$ Department of Biomedical Engineering, University of Texas at Austin, Austin, TX, USA. \\ ${ }^{5}$ Department of Biomedical Engineering, Washington University in St. Louis, St. Louis, MO, USA. \\ ${ }^{6}$ Department of Biomedical Engineering, University of Texas at Austin, Austin, TX, USA. \\ ${ }^{7}$ Gladstone Institutes, San Francisco, CA, USA. \\ ${ }^{8}$ Department of Bioengineering and Therapeutic Sciences, University of California, San Francisco, \\ San Francisco, CA, USA.
}

\section{Abstract}

V2a interneurons are located in the hindbrain and spinal cord where they provide rhythmic input to major motor control centers. Many of the phenotypic properties and functions of excitatory V2a interneurons have yet to be fully defined, which could lead to novel regenerative therapies for traumatic injuries and drug targets for chronic degenerative diseases. Here, we describe how to produce V2a interneurons from mouse and human pluripotent stem cells (PSCs), as well as strategies to characterize and mature the cells for further analysis. The described protocols are based on a sequence of small molecule treatments to induce differentiation into V2a interneurons. We also include a detailed description of how to phenotypically characterize, mature, and freeze the cells. The mouse and human protocols are similar in the sequence of small molecules used, but

\footnotetext{
*Corresponding Author sakiyama@utexas.edu, Todd.mcdevitt@gladstone.ucsf.edu. AUTHOR CONTRIBUTIONS

J.C.B designed, performed and analyzed human and mouse differentiation experiments. N.R.I, N.W., and R.T. designed, performed and analyzed mouse differentiation experiments. S.S.E designed the mouse differentiation experiments. T.C.M designed the human differentiation experiments. J.C.B, N.R.I, S.S.E and T.C.M prepared the manuscript.

COMPETING FINANCIAL INTERESTS

The authors declare no competing interests.

DATA AVAILABILITY

Data are available from the authors upon request.

EDITORIAL SUMMARY V2a interneurons are differentiated from mouse and human pluripotent stem cells following culture in the presence of a sequence of small molecule treatments.

TWEET Differentiation of V2a interneurons from human and mouse pluripotent stem cells @ JessicaCButts @ToddMcDevitt @DrSakiyama@GladstoneInst

COVER TEASER Production of V2a interneurons
} 
differ slightly in the concentrations and durations necessary for induction. Based on the protocols described, scientists can expect to obtain V2a interneurons with a purity of $\sim 75 \%$ in 7 days and $\sim 50 \%$ in 20 days from mouse and human PSCs, respectively.

\section{Keywords}

V2a interneurons; Human pluripotent stem cells; Mouse pluripotent stem cells; stem cell differentiation; neuroscience; PSC; mPSC; hPSC; stem cell

\section{INTRODUCTION}

Interneurons in the ventral half of the brainstem and spinal cord are critical to the coordination of a wide variety of motor control tasks. A delicate balance of excitatory and inhibitory inputs from interneurons onto motor control centers is essential for proper autonomic and voluntary motor function. In the brainstem, V2a interneurons located in the medial reticular formation of the hindbrain provide rhythmic excitatory input to the PreBötzinger complex, which contributes to regulation of respiration ${ }^{1,2}$. V2a interneurons in the spinal cord have been identified throughout the rostral-caudal axis and are suspected to provide rhythmic input to excitatory commissural interneuron subtypes (V0 and V3) and caudal motor neurons ${ }^{3,4}$. Following a disruptive event to the central nervous system, through spinal cord injury or neurodegenerative disease, such as ALS, interneuron connections are disrupted, leading to loss of motor control ${ }^{5,6}$. Further, V2a interneurons have been implicated as an important compensatory mechanism to facilitate respiration following onset of ALS and after cervical spinal cord injury ${ }^{5,7}$. We have previously described the differentiation of V2a interneurons from mouse ${ }^{8,9}$ and human PSCs ${ }^{10}$. Here we present a detailed protocol focused on how to derive glutamatergic V2a interneurons from murine and human pluripotent stem cells (PSCs).

\section{Applications of the protocol}

The ability to derive V2a interneurons from pluripotent sources will enable further examination of the phenotypic properties of these specific excitatory interneurons and could be exploited for disease modeling and therapeutic applications. In vitro, V2a interneurons can be used to investigate mechanisms of spinal neural development and maturation of PSCderived neurons ex vivo, in addition to examining neural circuit formation and synaptogenesis with other cells, such as motor neurons. The purified or enriched populations of V2a interneurons can also be transplanted into different regions of the spinal cord to examine how they integrate within a native tissue setting ${ }^{7}$. The therapeutic potential of the cells can be examined following transplantation to injured or diseased CNS tissue in order to assess the potential functional recovery of augmenting V2a interneurons that might bridge damaged tissue regions and re-established functional synapse connections with endogenous cells of impaired motor circuits.

\section{Development of the protocol}

The V2a interneuron protocols from mouse and human PSCs were inspired by the critical signaling morphogens present during embryonic development of the primitive neural tube. 
Comparative studies of mouse and human development have demonstrated that the same transcription factors drive similar phenotypic pattern generation during neural tube formation between the two species ${ }^{11}$. The key signaling molecules that are necessary to induce V2a interneuron differentiation are retinoic acid (RA), a Sonic Hedgehog (Shh) agonist, and a $\gamma$-secretase inhibitor to impede Notch signaling. During neural tube formation, RA is released from adjacent somites along the spinal cord where signaling is highest in the cervical region, and gradually decreases in both the rostral and caudal directions ${ }^{12}$. Addition of RA during differentiation promotes a more caudal neural phenotype that correlates to where V2a interneurons naturally arise ${ }^{13}$. During development, Shh is released from the floor plate and notochord, to produce a ventral-dorsal gradient that spatially patterns the progenitor domains from which motor neurons and interneurons involved in motor control coincidentally emerge ${ }^{14}$. In the described protocol, purmorphamine (pur), a smoothened agonist, is used to activate the Shh pathway and induce a ventral neuronal phenotype. During neurogenesis, Notch signaling maintains a neural progenitor phenotype while the absence of Notch promotes neuronal differentiation ${ }^{15-17}$. Moreover, Notch inhibition specifies the V2a phenotype over the V2b phenotype from the common $\mathrm{p} 2$ progenitor ${ }^{18}$. Therefore, a critical element of the V2a interneuron protocol is the addition of the $\gamma$-secretase inhibitor N-[N-(3,5-Difluorophenacetyl)-L-alanyl]-Sphenylglycine t-butyl ester (DAPT) to inhibit Notch signaling and promote the specification of V2a interneurons. The combination of RA, pur, and DAPT can be used to induce V2a interneurons from mouse and human PSCs (Fig. 1).

Our previous reports provided the first descriptions of the differentiation of V2a interneurons from mouse ${ }^{8,9}$ and human PSCs ${ }^{10}$. In Brown et al. and Butts et al., the concentrations of RA, pur, and DAPT were determined by sequentially varying individual molecules to identify the respective concentration(s) that resulted in the highest percentage of cells expressing Ceh-10 Homeodomain-Containing Homolog (CHX10, also known as VSX2), a defining marker of V2a interneurons in the hindbrain and spinal cord. The addition of DAPT following neuronal specification is used to inhibit Notch signaling in order to promote the excitatory V2a subtype instead of inhibitory V2b interneurons.

\section{Comparison with other methods to differentiate neural cells.}

Although several protocols have been described for differentiation of neural cells from PSCs, our protocols for V2a interneuron differentiation were the first published methods to produce these cells. Compared to motor neuron induction protocols from PSCs ${ }^{19,20}$, the V2a interneuron differentiation requires a lower concentration of retinoic acid and a lower concentration of the Shh agonist, pur, to specify a more dorsal population of interneurons. To date, directed differentiation protocols for V3 and V1 interneurons have only been described from mouse $\mathrm{PSCs}^{21-23}$. Compared to mouse V2a differentiation, generation of V3 interneurons requires two additional days of induction with a stronger Shh agonist (smoothened agonist, SAG), which reflects endogenous Shh signaling (Fig. 1) ${ }^{22,23}$. On the other hand, very low levels $(0.5 \mathrm{nM}-5 \mathrm{nM})$ of SAG are used to induce the developmentally more dorsal V1 interneurons (Fig. 1) ${ }^{21,22}$. Additional protocols for specific neural subtypes including glia, motor neurons, as well as ventral and dorsal interneurons have been comprehensively reviewed by White et $\mathrm{al}^{24}$. This protocol for V2a interneurons describes 
how to induce committed V2a interneurons from mouse PSCs in 6 days and from human PSCs in 17 days. In addition, we describe how to enrich, mature, characterize and freeze committed V2a interneuron populations. The comparable protocols reliably yield a population of $\mathrm{V} 2 \mathrm{a}$ interneurons from both species that enables further investigation of this excitatory neuronal population that is critical to various motor and autonomic activities.

Limitations of this protocol.-Despite our initial success differentiating V2a interneurons from PSCs, several limitations exist to the current methods and the derived cells. There can be batch-to-batch variability in the yield of V2a interneurons with percentages ranging sometimes as low as $10 \%$ to upwards of $50 \% \mathrm{CHX}^{+} 0^{+}$cells. To avoid low yields, it is important to keep track of specific timing of media changes and small molecule additions. It is best to be consistent and perform cell culture within the same 1hour when doing the media changes. Another primary source of variability can arise from subtle shifts in the rate of differentiation between experiments and especially between different cell lines. For the human differentiation, CHX10 percentage is typically analyzed at a fixed time point of 17 days. However, the true peak percentage of CHX10 expression may vary slightly depending on the initial cell density, as well as growth and differentiation rate of the cells. Real-time monitoring of V2a differentiation using an endogenous reporter line would be one possible solution, however creation of such a line has been problematic to date due to low efficiency targeting of the CHX10 locus in hPSCs. One limitation is that in the absence of selection to purify the V2a interneurons, the derived cultures are a heterogeneous population. The non-V2a interneurons in the culture are neural but often their specific identities are not thoroughly characterized in every experiment. Additionally, there are several subtypes of committed $\mathrm{V} 2$ interneurons, which come from a common $\mathrm{p} 2$ progenitor, that are not currently characterized with this protocol including inhibitory GATA $3^{+} \mathrm{V} 2 \mathrm{~b}$ and $\mathrm{V} 2 \mathrm{c}$, and the excitatory $\mathrm{SHOX}^{+} / \mathrm{CHX}^{-} 0^{-} \mathrm{V} 2 \mathrm{~d}$ lineage ${ }^{18,25,26}$. If a particular subtype of V2 interneurons is of interest, additional experimentation and staining may be performed.

\section{Experimental Design}

The following protocol provides a step-by-step description of how to differentiate, enrich, mature, and analyze V2a interneurons from mouse and human pluripotent stem cells.

Differentiation of V2a interneurons.-An overview of the V2a interneuron differentiation protocol from both mouse and human PSCs is depicted in Figure 2. While there are subtle differences between the mouse and human protocols, each proceeds through the progressive stages of neuralization, caudalization, and V2a specification. To ensure the best chances of successful differentiation, a healthy and homogenous batch of pluripotent cells that exhibit a consistent growth rate and contain fewer than $10 \%$ differentiated cells should be used. When starting from a cryopreserved population of PSCs, the cells should be passaged a minimum of three times for post-thaw recovery and consistent growth before attempting to differentiate. The first step of the protocol is to direct the PSCs to a neural fate. In the mouse system, leukemia inhibitory factor is removed, and the medium is changed to promote neural differentiation (Fig. 2a). In the human system, neuralization is achieved with dual SMAD inhibition using the TGF- $\beta$ and BMP inhibitors, SB 435142 and LDN 193189, in mTeSR medium (Fig. 2b) ${ }^{27}$. RA is added during the next phase of the differentiation to 
caudalize the cell population. In the last phase of the differentiation, pur and DAPT are added to promote the emergence of $\mathrm{p} 2$ progenitors and stimulate V2a differentiation in lieu of a V2b fate, respectively. The induction of V2a interneurons can be enhanced in the mouse system by using a genetically modified cell line with a CHX10 driven selection cassette ${ }^{9}$ and in the human system with a replating step.

The V2a interneuron cultures undergo morphological changes throughout the differentiation, which can be visually monitored to ensure that the differentiation is progressing as expected. When beginning the differentiation, it is critical that the pluripotent cells are 70-80\% confluent and have less than $10 \%$ differentiated cells based upon visual inspection of epithelial-like cells migrating out of the pluripotent colonies (Fig. $3 a_{i}$ and $3 b_{i}$ ). For the mouse system, differentiation is initiated by a change to DFK5 medium (DMEM:F12 supplemented with knockout serum replacement (KSR)) as well as the spontaneous formation of embryoid bodies. The size of the aggregates is heterogeneous (approximately 75 to $225 \mu \mathrm{m}$ in diameter on day 2, Fig. $3 \mathrm{a}_{\mathrm{ii}}$ ) and the aggregates continue to grow throughout the differentiation (approximately 125 to $325 \mu \mathrm{m}$ in diameter on day 6, Fig. $3 \mathrm{a}_{\mathrm{iv}}$ ).

Alternatively, the embryoid bodies can be plated onto gelatin-coated well plates at day 2 of the differentiation to test different culture conditions in a high-throughput method. For the human system, the cells are approximately $50 \%$ confluent after one day of differentiation and are less compact compared to pluripotent colonies with protrusions at the colony edges due to the presence of the RHO/ROCK pathway inhibitor, Y-27632 (Fig. 3 $b_{i i}$ ). A fluorescent Lamin- $B_{1}:: m E G F P$ reporter can be used to identify nuclei morphologies that may not be readily apparent through phase imaging. By day 3 , the cultures become fully confluent and the cells begin to become more compact (arrowhead, Fig. $3 b_{i i i}$ ). On day 7 , the differentiation cultures have grown to be multiple layers thick and local areas of condensed, radially aligned nuclei can be observed (arrowheads, Fig. $3 b_{\text {iv }}$ ). By the end of the differentiation on day 17, the cell layers have become thick enough that it is difficult to discern any unique topology by phase imaging, although areas of more- and less-dense regions can be observed with the fluorescent nuclear lamina (arrowheads, Fig. $3 b_{v}$ ). Assessment of the quality of the progression through the differentiation process can be done by visual inspection. Deviation from cell density and/or cell morphology displayed in Figure 3 may suggest that an error has been made in the protocol. One example of irregular cell density is displayed in Supplemental Figure 1. On days 3 and 5, the cell layers look normal as a full confluent monolayer of cells covers the bottom of the well. However, by day 7, cells begin to detach in some areas of the plate (denoted by area marked with “*”). As the differentiation progresses to day 13, neurons are visible in the cell culture (inset (i), arrow head) but the efficiency of differentiation is reduced because dense monolayers were not formed. In this case, we identified that using freshly diluted Matrigel, less than 1 week old, prevented cell detachment.

Phenotypic Characterization of V2a Interneurons.-At the end of the differentiation, the V2a interneuron phenotype can be characterized by flow cytometry, immunocytochemistry and quantitative PCR. The described protocol is expected to yield CHX $10^{+}$cell percentages of $\sim 20 \%$ from mouse PSCs and 20-50\% from human PSCs depending on the cell line (Fig. 4a-b). The gating strategy used for this analysis is depicted 
in Figure 8. Using an antibody against CHX10, the V2a interneurons are readily visualized by fluorescence microscopy (Fig. 4c-d). For cytometry and immunostaining, proper isotype controls should be used to account for any non-specific background staining. qPCR can assess the increased gene expression of multiple markers of the V2a lineage (mouse: Forkhead Box N4 (Foxn4), Lim Homeobox 3 (Lhx3), and Chx10 and human: FOXN4, SRYBox 14 (SOX14), and CHX10) as well as suppression of motor neurons (Homeobox HB9 ( $H B 9$ also known as MNXI) and retinal cell types (Cone-Rod Homeobox (CRX)) because CHX10 is expressed by bipolar cells in the developing retina (Fig. 4e-f). These characterization steps are critical to confirming the specificity and efficiency of the differentiation process.

Enrichment of the V2a interneurons in a heterogeneous culture is achieved in the mouse system by using the Chx10::puro mESC cell line and applying antibiotic selection at day 6 to purify the V2a interneuron population to $\sim 80 \%$ (Fig. 5a). Treatment of differentiated wild type mESCs with antibiotic selection under the same conditions can serve as a negative control for the selection process when establishing an initial workflow. The human V2a interneuron population can be enriched by dissociating and replating the cultures at day 17 after initial commitment $\left(\mathrm{CHX10^{+ }}\right.$ ) and prior to analysis at day 20 (Fig. 5b-c). Replating can enrich the $\mathrm{CHX} 10^{+} \%$ up to 6 -fold when differentiation is low (Fig. 5b), but the effectiveness of the replating step is decreased when the differentiation efficiency is high (>50\%) by day 20 (Fig. 5c). Therefore, the relative effectiveness of the replating is inversely related to the efficiency of the initial differentiation efficiency, though replating always results in an improvement from the baseline differentiation condition. Replating does reduce the total number of cells available, so a researcher can individually decide if replating is necessary depending on the intended purpose(s) of an experiment.

Physiological Maturation of V2a interneurons.-The committed V2a interneurons begin to mature into functional neurons with additional long-term culture in maturation medium supplemented with growth factors. At early time points (day 20 for mouse and human), $\mathrm{CHX} 10^{+}$cells have little expression of vesicular glutamate transporter 2 (VGlut2), a key marker of glutamatergic neurons (Fig. $6 \mathrm{a}_{\mathrm{i}}$ and $6 \mathrm{~b}_{\mathrm{i}}$ ). To improve maturation and survival of purified mouse V2a interneurons for long periods of time, cells are co-cultured with purified Oligodendrocyte Transcription Factor $2(\mathrm{Olig} 2)^{+}$progenitors, which differentiate into predominantly glial populations. By day 34 for mouse and day 60 for human, the V2a interneuron cultures have abundant expression of VGlut2, indicating maturation of the differentiating cultures (Fig. $6 \mathrm{a}_{\mathrm{ii}}$ and $6 \mathrm{~b}_{\mathrm{ii}}$ ). Calcium imaging of V2a interneuron cultures can be used as a longitudinal proxy of electrical excitability and nascent sign of functional maturation. The selected mouse V2a interneurons mixed with pure Olig2 ${ }^{+}$cells aggregate for 48 hours before a 24-hour transfection with the AAV1-hSyn-GCaMP6f adeno-associated virus (AAV). Following two weeks of maturation, the mouse aggregates display synchronous bursts of calcium flux that can last approximately 2 seconds (arrowheads, Fig. $6 \mathrm{c}$ and Supplementary Video 1). To assess spontaneous calcium bursts in human V2a interneuron cultures, differentiation studies are performed with an iPSC cell line modified with a constitutive GCaMP6f. This experiment could be performed similarly using Fluo-4 AM or similar fluorescent calcium dyes. By 45 days of culture, neurons display spontaneous 
calcium activity (arrowheads, Fig. 6d and Supplementary Video 2), with calcium bursts observed in the soma and neurites that last approximately 4 seconds. Calcium imaging analysis confirms that the in vitro derived populations are functionally active.

Freezing of human V2a interneurons.-The duration of the human differentiation protocol (almost 3 weeks) can make logistical planning of long-term experiments difficult. Therefore, we examined how to freeze and thaw committed human V2a interneurons such that the differentiation protocol could be transiently suspended soon after definitive interneuron commitment to allow experiments and analyses to be performed at later time points (i.e. electrophysiological testing, synaptogenesis experiments, cell transplantation). V2a interneuron day 17 cultures can be dissociated and frozen in a mixture of 50\% neural induction medium, 40\% fetal bovine serum (FBS), and 10\% dimethyl sulfoxide (DMSO) solution then stored in liquid nitrogen until needed. Post-thaw, the V2a interneurons are cultured for at least 3 days to allow viable neurons to adhere before subsequent experiments take place. The percentage of $\mathrm{CHX} 10^{+}$cells is maintained through the cryopreservation process despite a $\sim 30 \%$ decrease in overall cell yield (Fig. 7a-b). The cultures maintain similar organization as observed through immunocytochemistry for CHX10 (Fig. 7c-d). The recovered V2a interneuron cell yield is a critical consideration for subsequent experimental planning to ensure that sufficient quantities of cells are available to perform maturation, transplantation, or other studies.

\section{MATERIALS}

\section{Reagents}

- $\quad$ Accutase (Accutase, cat. no. AT104)

- Agar (Fisher, cat. no. BP-1423)

- $\quad$ AggreWell Rinsing Solution (Stem Cell Technologies, cat. no. 07010)

- $\quad$ B-27 Supplement (Life Technologies, cat. no. 17504-044)

- $\quad$ BD Transcription factor buffer set containing $4 x$ fix perm buffer, diluent buffer, and 5x perm/was buffer (BD, cat. no. 562574)

- $\quad$ CAUTION!: Fixation/permeabilization buffer contains methanol follow proper chemical waste disposal procedures.

- $\quad \beta$-mercaptoethanol (BME, 55mM, Life Technologies, cat. no. 21985-023)

- $\quad$ CAUTION!: $\beta$-mercaptoethanol is corrosive, toxic if ingested, and a hazard to your health and the water supply. Wear proper PPE when using this reagent and dispose of properly.

- $\quad$ Bovine Serum Albumin (BSA, Sigma, cat. no. A3311)

- $\quad$ BrainPhys TM Neuronal Medium (Stem Cell Technologies, cat. no. 05790)

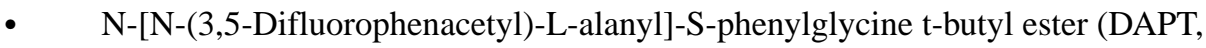
Sigma, cat. no. D5942-5MG)

- $\quad$ Dimethyl Sulfoxide (DMSO, Sigma, cat. no. D2650) 
- $\quad$ DNAse (1\%, Sigma, cat. no. DN25)

- Dulbecco's Modification of Eagle's Medium (DMEM, Life Technologies, cat. no. 11965-092)

- Dulbecco's Modification of Eagle's Medium/Ham's F-12 50/50 Mix (DMEM/ F12, Cellgro, cat. no. 10-090-CV)

- $\quad$ Dulbecco's Phosphate Buffered Saline (PBS, Life technologies, cat. no. 14190144)

- $\quad$ Fast SYBR green master mix (Thermo Fisher, cat. no. 4385612)

- $\quad$ Fetal Bovine Serum (FBS, Atlanta Biologicals, cat. no. S11150)

- $\quad$ Fluo-4, AM, cell permeant (Thermo Fisher, cat. no. F14201)

- $\quad$ Gelatin solution (2\%, Sigma, cat. no. G1393)

- $\quad$ GlutaMAX (Life Technologies, cat. no. 17504-044)

- $\quad$ Heparin ammonium salt from porcine intestinal mucosa (Sigma Aldrich, cat. no. H6279-1MU)

- $\quad$ HEPES-Buffered Saline Solution (HBSS, Sigma Aldrich, cat. no. 51558)

- $\quad$ HEPES (Life Technologies, cat. no. 15630-080)

- $\quad$ Hoechst 33342 (Thermo Scientific, cat. no. 62249)

- InSolution Purmorphamine (Millipore, cat. no. 540223)

- Insulin/Transferrin/Selenium (ITS, Life Technologies, cat. no. 41400-045)

- $\quad$ iScript cDNA kit (Bio-Rad, cat. no. 1708891)

- $\quad$ Knockout DMEM (Life Technologies, cat. no. 10829-018)

- $\quad$ Knockout replacement serum (KSR, Life Technologies, cat. no. 10828-028)

- $\quad$ L-Ascorbic acid 2-phosphate magnesium salt hydrate (AA, Sigma Aldrich, cat. no. A8960-5G)

- $\quad$ LDN-193189 (10 mM, Stemgent, cat. no. 04-0074-02)

- $\quad$ Laminin (1.18 mg/mL, Life Technologies, cat. no. 23017-015)

- Leukemia Inhibitory Factor (LIF, Millipore ESGRO, cat. no. ESG1106)

- L-Glutamine (200 mM, Corning, cat. no. 25-005-Cl)

- Matrigel Growth Factor Reduced Phenol Free Basement Membrane Matrix (Corning, cat. no. 35623)

- $\quad$ CRITICAL: The human differentiation is sensitive to the basement membrane the cells are grown on. This protocol has been established using Corning Matrigel. If the cells have been grown on a different basement membrane, slowly transition the cells to Matrigel over several 
passages. It is also critical that the diluted Matrigel is less than a week old before use.

- $\quad$ Matrigel hESC Qualified Matrix (Corning, cat. no. 354277)

- CRITICAL: For any ESC lines, it is important to use the hESC Qualified Matrigel.

- $\quad$ mTeSR1 (StemCell Technologies, cat. no. 05850)

- $\quad$ N2 supplement (Life Technologies, cat. no. 17501-048)

- $\quad$ Neurobasal (Life Technologies, cat. no. 21103-049)

- $\quad$ NeuroCult ${ }^{\mathrm{TM}}$ SM1 Neuronal Supplement (Stem Cell Technologies, cat. no. 05711)

- $\quad$ Newborn Calf Serum (NBCS, Life Technologies, cat. no. 16010-167)

- Nonessential Amino Acids (100X, Corning, cat. no. 25-025-Cl)

- $\quad$ Normal Donkey Serum (NDS, Jackson ImmunoResearch, cat. no. 017-000-121)

- $\quad$ Nucleosides (100X, Millipore, cat. no. ES-008-D)

- $\quad$ Paraformaldehyde (16\% w/v, VWR, cat. no. AA43368-9M)

- CAUTION!: Paraformaldehyde is a carcinogen and an irritant. Wear proper PPE when using this reagent and dispose of properly.

- Penicillin Streptomycin (100X, Corning, cat. no. 30-002-Cl)

- $\quad$ Phosphate Buffered Saline 10X Molecular Biology Grade (Corning 46-013-CM)

- $\quad$ Poly-L-ornithine (0.01\%, Sigma, cat. no. P4957)

- $\quad$ Puromycin (Sigma, cat. no. P8833)

- $\quad$ Recombinant Human Brain Derived Neurotrophic Factor Protein (BDNF, R\&D Systems, cat. no. 248-BD-025)

- $\quad$ Recombinant Human Glial Derived Neurotrophic Factor Protein (GDNF, R\&D Systems, cat. no. 212-GD-010)

- Recombinant Human Ciliary Neurotrophic Factor Protein (CNTF, R\&D Systems, cat. no. 257-NT-010)

- Recombinant Human Insulin Growth Factor-1 Protein (IGF, R\&D Systems, cat. no. 291-G1-200)

- $\quad$ Recombinant Human Neurotrophin 3 (NT3, Peprotech, cat. no. 450-03)

- $\quad$ Retinoic Acid (50mg, Sigma, cat. no. R2625)

- CAUTION!: Acute toxicity. Open carefully in a chemical fume hood while wearing proper PPE

- $\quad$ RNeasy Mini kit (Qiagen, cat. no. 74106) 
- $\quad$ SB-435142 (10 mM, Stemgent, cat. no. 04-0010-05)

- $\quad T_{a q m a n}{ }^{\mathrm{TM}}$ Fast Advanced Master Mix (Thermo Fisher Scientific, cat. no. 4444556)

- $\quad$ Tris buffered saline $7.4 \mathrm{pH}$ (TBS, Fisher Scientific cat. no. BP24711)

- $\quad$ Triton $^{\mathrm{TM}} \mathrm{X}-100$ (Sigma, cat. no. T8787)

- CAUTION!: Triton is corrosive, an irritant, and hazardous to the water supply. Wear proper PPE when using this reagent and dispose of properly.

- $\quad$ Trypan blue ( $0.4 \%$, Thermo Fisher, cat. no. T10282)

- $\quad$ Trypsin-EDTA (0.25\%, Life Technologies, cat. no. 25200-072)

- $\quad$ AAV1-hSyn-GCAmp6f (Addgene, cat. no. 100837-AAV1)

- $\quad$ Y-27632 (ROCK inhibitor) 2HCL 50mg - Selleck Chemicals, cat. no. S1049

- $\quad$ Human pluripotent stem cell lines. We have successfully used H7 hESCs (WiCell, cat. no. WA07), Lamin-B 1 ::mEGFP iPSCs (Allen Institute, cat. no. AICS-0013), WTB iPSCs ${ }^{28}$, WTC10 iPSCs, WTC11 AAVS1::GCaMP6f iPSCs ${ }^{29}$, kind gifts from the lab of Dr. Bruce Conklin (More information at: https:// labs.gladstone.org/conklin/information-human-ips-cells.html)

- CAUTION!: These cell lines should be checked regularly for a normal karyotype and to ensure they are mycoplasma negative.

- $\quad$ Mouse embryonic stem cell lines. We have successfully used RW.4 (ATCC ${ }^{\circledR}$, cat. no. SCRC-1018 ${ }^{\mathrm{TM}}$ ), Olig2::Puro ${ }^{30}$, Chx10::Puro ${ }^{9}$, Chx10::Puro Rosa26::CAGTdTomato $^{9}$ (Created by the Sakiyama-Elbert Lab). Both human and mouse lines can be obtained from the authors of the cited publications.

\section{Equipment}

- $\quad 0.5 \mathrm{~mL}$ microcentrifuge tubes (VWR, cat. no. 89000-026)

- $\quad 1.5 \mathrm{~mL}$ microcentrifuge tubes (VWR, cat. no. 89000-028)

- $\quad 5 \mathrm{~mL}$ transport tubes (Fisher Scientific, cat. no. 02-681-132)

- $\quad 15 \mathrm{~mL}$ and $50 \mathrm{~mL}$ Falcon Tubes (Fisher Scientific, cat. nos. 430791 and 430829)

- $\quad$ Nalgene ${ }^{\circledR}$ Polycarbonate Straight-Sided Wide-Mouth Jars (VWR, cat. no. 16129-310)

- $\quad$ T25 flasks (Corning, cat. no. 430639)

- $\quad 10 \mathrm{~cm}$ dish (Fisher Scientific, cat. no. 08-772-32)

- $\quad \mu$-slide 8-well chamber slides (ibidi, cat. no. 80826)

- Cellstar multiwall culture plates; 24 well and 48 well (Sigma Aldrich, cat. nos. M8812 and M8937) 
- $\quad$ 96-well round bottom plate (Corning, cat. no. 3799)

- $\quad$ Round bottom flow tube with cell strainer (Corning, cat. no. 352235)

- $\quad$ AggreWell Plate (Stem Cell Technologies, cat. no. 27845)

- $\quad$ Sterile $0.2 \mu \mathrm{m}$ acrodisc syringe filters (Pall Corporation, cat. no. PN4192)

- $\quad 10 \mathrm{~mL}$ luer-lok disposable syringes (Fisher Scientific, cat. no. 14-823-2A)

- $\quad 500 \mathrm{~mL}$ stericup filtration system (Corning, cat. no. 430758)

- $\quad 1 \mathrm{~L}$ stericup filtration system (Corning, cat. no. 430186)

- Wide orifice p1000 tips (Rainin, cat. no. 17007099)

- $\quad$ Parafilm (Bemis, cat. no. PM996)

- $\quad$ Nalgene ${ }^{\circledR}$ “Mr. Frosty” freezing container (VWR, cat. no. 5100-0001)

- $\quad$ Cryogenic vials (Corning, cat. no. 431416)

- $\quad$ Mini Pro Bath (RevSci, cat. no. RS-PV-50)

- $\quad$ Vortex (VWR, cat. no. 97043-562)

- $\quad$ Countess II FL Automated Cell Counter (Thermo Fisher Scientific)

- $\quad$ Countess ${ }^{\mathrm{TM}}$ Cell Counting Chamber Slides (Thermo Fisher Scientific, cat. no. C10312)

- $\quad$ 5810R Centrifuge (Eppendorf, cat. no. 022628168)

- $\quad$ Microplate adapters (Eppendorf, cat. no. 022638068)

- Nanodrop 2000c (Thermo Fisher Scientific, cat. no. ND-2000C)

- $\quad$ Simpliamp Thermocycler (Life Technologies, cat. no. A24811)

- $\quad$ StepOnePlus ${ }^{\mathrm{TM}}$ Real-Time PCR System (Life Technologies Scientific, cat. no. 4376598)

- SterilGARD Biological safety cabinet (The Baker Company model, cat. no. SG403)

- $\quad$ Heracell 240i Humidified incubator (Thermo Fisher Scientific)

- $\quad$ Inverted fluorescent microscope (Zeiss, Axio Observer Z1)

- $\quad$ ORCA-Flash4.0 LT PLUS Digital CMOS camera (Hamamatsu, cat. no. C1144042U30)

- FlowJo V10 Software (FlowJo, https://www.flowjo.com/solutions/flowjo/ downloads)

- Zen Blue Software (Zeiss, https://www.zeiss.com/microscopy/int/products/ microscope-software/zen.html) 


\section{Reagent Setup}

- $\quad 0.1 \% \mathrm{vol} / \mathrm{vol}$ gelatin solution $(\mathbf{5 0 0} \mathrm{mL})$ : Place $2 \%$ gelatin solution in warm water bath. Add $25 \mathrm{~mL} 2 \%$ gelatin to $475 \mathrm{~mL}$ of deionized water. Sterile filter into $500 \mathrm{~mL}$ filter bottle. Store at $4^{\circ} \mathrm{C}$ for up to a month.

- $\quad \mathbf{0 . 1 \%} \mathbf{w t} / \mathbf{v o l}$ agar solution $(\mathbf{2 0 0} \mathbf{~ m L})$ : Add $0.2 \mathrm{~g}$ agar to $200 \mathrm{~mL}$ of deionized water. Autoclave on liquid cycle to sterilize. Store at room temperature (approximately $22^{\circ} \mathrm{C}$ in our laboratory) for up to 1 year.

- $\quad 4 \% \mathrm{wt} / \mathrm{vol}$ Paraformaldehyde $(\mathbf{4 0} \mathrm{mL})$ : Dilute $10 \mathrm{~mL}$ ampule of $16 \%$ paraformaldehyde in $30 \mathrm{~mL} 1 \mathrm{X}$ PBS. Store at $4^{\circ} \mathrm{C}$ for up to a month.

- 1X Phosphate buffered saline (1 L): Dilute $100 \mathrm{~mL}$ 10X PBS into $900 \mathrm{~mL}$ deionized water. Store at room temperature for up to a month.

- $\quad \mathbf{0 . 1 \%}$ vol/vol Triton $(500 \mathrm{~mL})$ : For a $500 \mathrm{~mL}$ final volume, add $500 \mu \mathrm{L}$ TritonX 100 to $500 \mathrm{~mL} 1 \mathrm{X}$ PBS. Store at room temperature for up to a month.

- $\quad$ Normal donkey serum $(\mathbf{1 0} \mathbf{~ m L})$ : Add $10 \mathrm{~mL}$ deionized water to the vial containing the lyophilized powder. Carefully swirl until the powder is completely dissolved. Aliquot into smaller working volumes $(0.5$ to $1 \mathrm{~mL})$ to mitigate freeze-thaw cycles. Store at $-20^{\circ} \mathrm{C}$ for up to 1 year.

- $\quad$ Fixation/permeabilization buffer: Dilute 1 part fix/perm buffer into 3 parts diluent buffer. Keep cold before use. This solution can be stored at $4^{\circ} \mathrm{C}$ for up to a week.

- Permeabilization/wash buffer: Dilute 1 part perm/wash buffer into 4 parts deionized water. Keep on ice during use. This solution can be stored at $4{ }^{\circ} \mathrm{C}$ for up to a week.

- $\quad$ Aliquot Matrigel: Thaw container of Matrigel at $4{ }^{\circ} \mathrm{C}$ overnight. Chill autoclaved p1000 pipette tips and $1.5 \mathrm{~mL}$ microcentrifuge tube tubes at $-20^{\circ} \mathrm{C}$ overnight. For hESC-qualified Matrigel, identify the suggested diluted volume specific to the lot on the specification sheet from the manufacturer. For growth factor reduced (GFR) Matrigel, identify the protein concentration on the specification sheet and aliquot the volume that when diluted into $50 \mathrm{~mL}$ will result in a final concentration of $80 \mu \mathrm{g} / \mathrm{mL}$. Sterilely aliquot the specified volume of thawed Matrigel into $1.5 \mathrm{~mL}$ microcentrifuge tubes that are on ice. Store at $-20^{\circ} \mathrm{C}$ until manufacturer's expiration date.

- Dilute Matrigel (50mL): Place a $50 \mathrm{~mL}$ conical containing $50 \mathrm{~mL}$ cold knock out DMEM, a p1000 pipette and tips in a biosafety cabinet. Quickly bring one aliquot of Matrigel from the $-20^{\circ} \mathrm{C}$ to the biosafety cabinet. Pipette $1 \mathrm{~mL}$ of knockout DMEM and thaw the Matrigel by pipetting the knockout DMEM up and down in the microcentrifuge tube. Expend contents into the $50 \mathrm{~mL}$ conical tube. Repeat this process until the Matrigel is fully thawed. Rinse out the microcentrifuge tube with the knockout DMEM to ensure all of the Matrigel has been incorporated. Store diluted Matrigel at $4^{\circ} \mathrm{C}$ for 1 week, but best if used within a few days. 
- $\quad$ Aliquot L-Glutamine: Thaw and fully re-suspend 200mM L-glutamine stock solution. Ensure the solids are fully dissolved Aliquot into $5 \mathrm{~mL}$ aliquots. Store at $-20^{\circ} \mathrm{C}$ until manufacturer's expiration date.

- Aliquot penicillin/streptomycin: Thaw and aliquot the 100X penicillin/ streptomycin stock solution into $5 \mathrm{~mL}$ aliquots. Store at $-20^{\circ} \mathrm{C}$ until manufacturer's expiration date.

- $\quad 0.1 \% \mathrm{wt} / \mathrm{vol}$ bovine serum albumin (BSA) in PBS: Add $10 \mathrm{mg}$ BSA to a 15 $\mathrm{mL}$ conical. In a sterile environment, add $10 \mathrm{~mL}$ PBS and invert until the BSA has dissolved. Sterile filter using a $0.22 \mu \mathrm{m}$ filter and a $10 \mathrm{~mL}$ syringe. Store at $4^{\circ} \mathrm{C}$ for up to 1 week.

- InSolution Purmorphamine (pur): Thaw and aliquot into $15 \mu \mathrm{L}$ volumes. Store at $-20^{\circ} \mathrm{C}$ for up to 6 months.

- SB-435142: Thaw and aliquot into $100 \mu \mathrm{L}$ volumes. Store at $-20^{\circ} \mathrm{C}$ for up to 6 months.

- LDN-193189: Thaw and aliquot into $15 \mu \mathrm{L}$ volumes. Store at $-20^{\circ} \mathrm{C}$ for up to 6 months.

- $\quad$ Y-27632 - Rock inhibitor (10mM): Reconstitute $50 \mathrm{mg}$ in $15.61 \mathrm{~mL}$ DPBS for a $10 \mathrm{mM}$ solution. Aliquot into $500 \mu \mathrm{L}$ volumes. Store at $-20^{\circ} \mathrm{C}$ for up to 6 months. Once thawed, store at $4^{\circ} \mathrm{C}$ for two weeks.

- $\quad$ DAPT (10mM): Add $1.16 \mathrm{~mL}$ DMSO directly to the vial containing $5 \mathrm{mg}$ DAPT. Aliquot into $25 \mu \mathrm{L}$ volumes. Store at $-20^{\circ} \mathrm{C}$ for for up to 6 months. Once thawed, store at $4^{\circ} \mathrm{C}$ for one week.

- $\quad$ Retinoic Acid (RA, 20mM): In a chemical fume hood, crack open vial containing 50mg RA. Add $1 \mathrm{~mL}$ DMSO to the vial containing RA powder to resuspend. Transfer the $1 \mathrm{~mL}$ of RA solution to a $15 \mathrm{~mL}$ conical and bring the final volume up to $8.3 \mathrm{~mL}$ with DMSO. Make sure the solution is fully resuspended. In a sterile hood, sterile filter the solution through a $0.22 \mu \mathrm{m}$ filter. Aliquot into $20 \mu \mathrm{L}$ volumes and $1 \mathrm{~mL}$ volumes. Store the $20 \mu \mathrm{L}$ aliquots at $-20^{\circ} \mathrm{C}$ for up to 6 months. Store the $1 \mathrm{~mL}$ aliquots at $-80^{\circ} \mathrm{C}$ for up to 3 years.

- Heparin $(2 \mathrm{mg} / \mathrm{mL})$ : Measure $20 \mathrm{mg}$ heparin ammonium salt into a $15 \mathrm{~mL}$ conical, add $10 \mathrm{~mL}$ PBS, and sterile filter through a $0.22 \mu \mathrm{m}$ filter. Aliquot into $550 \mu \mathrm{L}$ volumes. Store at $-20^{\circ} \mathrm{C}$ for up to 6 months.

- $\quad$ Ascorbic Acid (AA, 5 mg/mL): Measure $5 \mathrm{mg}$ AA into a $15 \mathrm{~mL}$ conical, add 1 $\mathrm{mL}$ tissue culture grade water, and sterile filter through a $0.22 \mu \mathrm{m}$ filter. Aliquot into $50 \mu \mathrm{L}$ volumes. Store at $-20^{\circ} \mathrm{C}$ for up to 6 months.

- $\quad$ BDNF (25 $\mu \mathrm{g} / \mathrm{mL})$ : Reconstitute $25 \mu \mathrm{g}$ with $1 \mathrm{~mL}$ sterile $0.1 \%$ BSA. Aliquot into $25 \mu \mathrm{L}$ volumes. Store at $-20^{\circ} \mathrm{C}$ for 6 months Once thawed, store at $4^{\circ} \mathrm{C}$ for one week. 
- $\quad$ GDNF $(100 \mu \mathrm{g} / \mathrm{mL})$ : Reconstitute $10 \mu \mathrm{g}$ with $100 \mu \mathrm{L}$ sterile $0.1 \%$ BSA. Aliquot into $25 \mu \mathrm{L}$ volumes. Store at $-20^{\circ} \mathrm{C}$ for 6 months Once thawed, store at $4^{\circ} \mathrm{C}$ for one week.

- $\quad$ CNTF $(\mathbf{1 0 0} \mu \mathrm{g} / \mathrm{mL})$ : Reconstitute $10 \mu \mathrm{g}$ with $100 \mu \mathrm{L}$ sterile $0.1 \%$ BSA. Aliquot into $25 \mu \mathrm{L}$ volumes. Store at $-20^{\circ} \mathrm{C}$ for 6 months Once thawed, store at $4{ }^{\circ} \mathrm{C}$ for one week.

- $\quad$ IGF $(200 \mu \mathrm{g} / \mathrm{mL})$ : Reconstitute $200 \mu \mathrm{g}$ with $1 \mathrm{~mL}$ sterile $0.1 \%$ BSA. Aliquot into $25 \mu \mathrm{L}$ volumes. Store at $-20^{\circ} \mathrm{C}$ for 6 months Once thawed, store at $4^{\circ} \mathrm{C}$ for one week.

- Puromycin $(\mathbf{1 0 m g} / \mathbf{m L})$ : Reconstitute $10 \mathrm{mg}$ with $1 \mathrm{~mL}$ sterile water. Aliquot into $25 \mu \mathrm{L}$ volumes. Store at $-20^{\circ} \mathrm{C}$ for up to 6 months.

- $\quad$ Diluted Fluo-4 AM (5mM): Reconstitute $50 \mu \mathrm{g}$ with $9.1178 \mu \mathrm{L}$ of DMSO. Store at $-20^{\circ} \mathrm{C}$ for up to 2 weeks.

- $\quad$ mTeSR (500mL): Add $100 \mathrm{~mL}$ of mTeSR supplement to $400 \mathrm{~mL}$ bottle of mTeSR base media. Store at $4^{\circ} \mathrm{C}$ for 2 weeks.

- $\quad$ Neural Induction Media (NIM, 520.5 mL): Add 5 mL N2, 5 mL $200 \mathrm{mM} \mathrm{L-}$ Glutamine, $5 \mathrm{~mL}$ 100-X non-essential amino acids, $5 \mathrm{~mL}$ 100-X penicillin/ streptomycin, and $500 \mu \mathrm{L}$ heparin to a $500 \mathrm{~mL}$ bottle of DMEM F:12. Sterile filter the solution through a $500 \mathrm{~mL}$ filter bottle. Store at $4^{\circ} \mathrm{C}$ for 2 weeks.

- $\quad$ Brain Phys + SM1 (510 mL): Add $10 \mathrm{~mL}$ SM1 to a $500 \mathrm{~mL}$ bottle of BrainPhys ${ }^{\mathrm{TM}}$. Store at $4^{\circ} \mathrm{C}$ for 2 weeks. This can be scaled down if the media will not get used in 2 weeks. The SM1 can be aliquoted into smaller volumes and store at $-20^{\circ} \mathrm{C}$ until manufacturer's expiration date.

- $\quad$ Complete media $(605 \mathrm{~mL})$ : Add $50 \mathrm{~mL}$ FBS, $50 \mathrm{~mL} \mathrm{NBCS}$ and $5 \mathrm{~mL} 100 \mathrm{X}$ nucleosides to a $500 \mathrm{~mL}$ bottle of DMEM. Run through a $1 \mathrm{~L}$ stericup filtration system. Store at $4^{\circ} \mathrm{C}$ for up to 1 month.

- $\quad$ DFK5 Media (500 $\mathbf{~ m L})$ : Remove $35 \mathrm{~mL}$ from a $500 \mathrm{~mL}$ bottle of DMEM/F12. Add $25 \mathrm{~mL}$ KSR, $5 \mathrm{~mL}$ ITS, $2.5 \mathrm{~mL}$ 100X nucleosides, $2.5 \mathrm{~mL}$ 100X nonessential amino acids and $909 \mu \mathrm{L} 55 \mathrm{mM}$ BME. Run through $500 \mathrm{~mL}$ stericup filtration system. Store at $4^{\circ} \mathrm{C}$ for up to 1 month.

- Wash Media (512.5 mL): Add $12.5 \mathrm{~mL}$ HEPES to a $500 \mathrm{~mL}$ bottle of DMEM. Run through $500 \mathrm{~mL}$ stericup filtration system. Store at $4^{\circ} \mathrm{C}$ for up to 1 month.

- $\quad$ DFK5NB (50 mL): Mix $25 \mathrm{~mL}$ DFK5 with $25 \mathrm{~mL}$ neurobasal. Store at $4^{\circ} \mathrm{C}$ for up to 1 month.

- V2a Selection media: To DFK5NB media add B-27 supplement, glutaMAX, and 2 or $4 \mu \mathrm{g} / \mathrm{mL}$ puromycin. Do not store. Make fresh every time. 


\section{Equipment Setup}

- Matrigel coat plates: Add diluted Matrigel to wells of a tissue culture plate. Use $500 \mu \mathrm{L}$ in each well of a 24 -well plate, $250 \mu \mathrm{L}$ in each well of a 48 -well plate, $150 \mu \mathrm{L}$ ibidi chamber slide, and $3 \mathrm{~mL}$ in a T-25 flask. Incubate for a minimum of 24 hours at $37^{\circ} \mathrm{C}$. Plates can be stored in the incubator for up to 5 days as long as the plate does not dry out.

- $\quad$ Gelatin coat flasks and 24-well plates (makes 10, T25 flasks): Aliquot $50 \mathrm{~mL}$ of $0.1 \%$ gelatin solution into a $50 \mathrm{~mL}$ conical. Incubate at $37^{\circ} \mathrm{C}$ for 20 minutes. Sterilely add $5 \mathrm{~mL}$ gelatin solution to each T25 flask. For 24-well plates, add 1 $\mathrm{mL}$ of gelatin solution to each well. Store at room temperature for 24 hours or at $37^{\circ} \mathrm{C}$ for 1 hour. Aspirate off the gelatin solution and store at room temperature to dry for at least 24 hours before use. Can be stored at room temperature for up to a month.

- Agar coat plates: Warm the $0.1 \%$ agar solution in a microwave until the solution is a clear liquid. Microwave in short 10 to 15 second bursts to avoid boiling. To coat several plates at one time, add $10 \mathrm{~mL}$ of $0.1 \%$ agar solution to a $10 \mathrm{~cm}$ petri dish then remove the agar solution and place it into the next $10 \mathrm{~cm}$ dish. Swirl the solution around so that the whole surface is covered. Repeat until all dishes are coated. Keep the dishes at room temperature for 24 hours before use. Store at room temperature for up to two weeks.

- $\quad$ Laminin coat plates: Dilute $30 \mu \mathrm{L}$ of laminin stock in $3 \mathrm{~mL}$ of sterile HBSS to make a $0.01 \mathrm{mg} / \mathrm{mL}$ solution. Add $300 \mu \mathrm{L} 0.01 \%$ poly-l-ornithine solution to each well of a 24-well plate and incubate for 1 hour at $37^{\circ} \mathrm{C}$. Aspirate poly-1ornithine and wash 5 times with $1 \mathrm{~mL}$ TBS $(7.4 \mathrm{pH})$ making sure the wells are completely drained before proceeding. Add $200 \mu \mathrm{L}$ of diluted laminin solution to each well and incubate at $4^{\circ} \mathrm{C}$ for 24 hours. Wash once with $1 \mathrm{~mL}$ sterile HBSS. Remove HBSS right before plating the cells. Laminin-coated plates are best used fresh but can be stored at $4^{\circ} \mathrm{C}$ for 1 week.

- Flow cytometry data analysis: We collect flow cytometry data on a BD LSRII (mouse) and a BD Accuri ${ }^{\mathrm{TM}} \mathrm{C} 6$ (human) and analyze using FlowJo as show in Fig. 8. A minimum of 10,000 events should be collected for all experiments. For mouse samples, the cell population should be gated using Hoechst (Fig. 8a). For human samples, cells should be gated using side scatter area (SSC-A) vs forward scatter area FSC-A (Fig. 8c). Select single cells by gating the population that falls within a linear line $(\mathrm{y}=\mathrm{x})$ on the forward scatter height $(\mathrm{FSC}-\mathrm{H})$ vs FSC-A (Fig. 8d). At least 5,000 events should be analyzed for all experiment after nuclei or single cell gating. For both mouse and human samples, cells that stain positive for $\mathrm{CHX} 10^{+}$are assessed by setting the graph to the stained filter vs. FSC-A and selecting the population that is positive compared to the non-stained control (Fig. $8 \mathrm{~b}$ and e). The Layout Editor can be used to create the graphs. 


\section{PROCEDURE}

\section{Maintenance of PSCs - TIMING: Variable}

1) Maintain mouse ESC as described in option A and human ESC or iPSC as described in option B.

A. Maintenance of mouse ESCs (mESCs) - TIMING: variable

i. $\quad$ Seed $\sim 2.5$ million mESCs on a gelatin-coated T25 flask in 5 $\mathrm{mL}$ Add Complete Medium supplemented with $1000 \mathrm{U}$ per $\mathrm{mL}$ of leukemia inhibitory factor and $100 \mu \mathrm{M} \beta$ Mercaptoethanol (BME). Incubate at $37^{\circ} \mathrm{C}$.

ii. Inspect the cells daily and move on to passage the cells (as described in the following steps) once colonies are 70\% confluent, which is typically after 2 days when cells are passaged at a ratio of 1:5.

?Troubleshooting: 1A(ii)

iii. To passage, wash once with $1 \mathrm{~mL}$ Wash Medium. Add $1 \mathrm{~mL}$ of warmed $0.25 \%$ Trypsin-EDTA and incubate for 5 minutes at $37^{\circ} \mathrm{C}$.

iv. Quench the enzyme with a volume of Complete Medium equal to 4 times the amount of Typsin-EDTA. Pipette up and down to dislodge cells from the bottom of the flask.

v. For a 1:5 passage, add $1 \mathrm{~mL}$ of diluted cell solution to $4 \mathrm{~mL}$ of Complete Medium containing $5 \mu \mathrm{L}$ LIF ( 1 million U/mL stock) and 9.09 $\mu \mathrm{L}$ BME (55 mM stock) in a fresh gelatin coated flask.

B. Maintenance of human ESCs and iPSCs - TIMING: variable

i. Plate $250,000 \mathrm{hPSCs}$ onto a Matrigel-coated T25 flask in 5 $\mathrm{mL}$ mTeSR supplemented with $10 \mu \mathrm{M} \mathrm{Y-27632.} \mathrm{Incubate} \mathrm{at}$ $37^{\circ} \mathrm{C}$.

ii. Replace $5 \mathrm{~mL}$ mTeSR media daily until colonies are $70 \%$ confluent, which is typically 3 to 4 days depending on the cell line. Once cells are $70 \%$ confluent proceed to the next step to passage cells.

?Troubleshooting: 1B(ii)

iii. To passage, wash once with $3 \mathrm{~mL}$ PBS. Add $3 \mathrm{~mL}$ of Accutase and incubate for 5 minutes at $37^{\circ} \mathrm{C}$.

iv. Gently tilt the flask to ensure that all cells have released. Dilute the Accutase with $5 \mathrm{~mL}$ PBS and pipette up and down in the flask to fully dissociate the colonies. Transfer contents 
to a $15 \mathrm{~mL}$ conical tube. Centrifuge at $200 \mathrm{x}$ g for 5 minutes at room temperature.

v. Aspirate supernatant and resuspend cell pellet in $2.5 \mathrm{~mL}$ mTeSR containing $10 \mu \mathrm{M}$ Y-27632.

vi. To count cells, take $10 \mu \mathrm{L}$ of the cell suspension and thoroughly mix with $10 \mu \mathrm{L}$ of $0.4 \%$ trypan blue. Transfer 10 $\mu \mathrm{L}$ of the stained cells to a counting slide and count cells manually with a hemocytometer or automatically with a Countess $^{\mathrm{TM}}$. Calculate the number of cells per $\mathrm{mL}$ in the cell suspension.

vii. Replate $250,000 \mathrm{hPSCs}$ onto a Matrigel-coated T-25 flask with $5 \mathrm{~mL}$ mTeSR supplemented with $10 \mu \mathrm{M}$ Y-27632. Incubate at $37^{\circ} \mathrm{C}$.

- Critical Step: Passage cells at least three times out of thaw before use in a differentiation. This allows for the cells to recover and reach a normal growth rate.

\section{Differentiation of V2a Interneurons - TIMING: 6 days for mouse, 17 for human}

2) Differentiate cells as described in option A for mouse cells or option B for human cells.

A. Mouse V2a IN differentiation - TIMING: 6 days

i. Dissociate $70 \%$ confluent $\mathrm{mESCs}$ by washing once with $1 \mathrm{~mL}$ Wash Medium. Add $1 \mathrm{~mL}$ of $0.25 \%$ warmed Trypsin-EDTA and incubate for 5 minutes at $37^{\circ} \mathrm{C}$. Quench Trypsin-EDTA with $4 \mathrm{~mL}$ Complete Medium and count the cells.

ii. Calculate the volume necessary to seed $1 \times 10^{6}$ cells per 10 $\mathrm{cm}$ petri dish. Add the cell suspension to a new $15 \mathrm{~mL}$ conical and centrifuge at $200 \mathrm{xg}$ for 5 minutes at room temperature.

iii. Resuspend dissociated mESCs into $10 \mathrm{~mL}$ of DFK5 media in an agar-coated $10 \mathrm{~cm}$ petri dish. Incubate at $37^{\circ} \mathrm{C}$. This corresponds to day 0 of the differentiation.

iv. On day 2, prepare the differentiation media consisting of 10 mL DFK5 supplemented with $10 \mathrm{nM} \mathrm{RA}$ and $1 \mu \mathrm{M}$ pur. In a $1.5 \mathrm{~mL}$ centrifuge tube, dilute $1 \mu \mathrm{L}$ of $20 \mathrm{mM}$ stock RA into $999 \mu \mathrm{L}$ of DFK5 to make a 1:1000 working dilution. In a separate $0.5 \mathrm{~mL}$ centrifuge tube, dilute $1 \mu \mathrm{L}$ of $50 \mathrm{mM}$ stock pur into $99 \mu \mathrm{L}$ of DFK5 to make a 1:100 working dilution. To $10 \mathrm{~mL}$ DFK5, add $5 \mu \mathrm{L} \mathrm{1:1000} \mathrm{RA} \mathrm{dilution} \mathrm{and} 20 \mu \mathrm{L} \mathrm{1:100}$ pur dilution.

v. Collect the media plus cell aggregates from the agar plate using a $10 \mathrm{~mL}$ stripette and dispense into a $15 \mathrm{~mL}$ conical 
tube. Allow the aggregates to settle to the bottom for 5-10 minutes. While the aggregates are settling, add $9 \mathrm{~mL}$ fresh differentiation media to the $10 \mathrm{~cm}$ agar dish.

vi. Once the aggregates have settled, fully aspirate the old media and resuspend the pellet with the remaining $1 \mathrm{~mL}$ of the differentiation media and add to the $10 \mathrm{~cm}$ dish. Incubate at $37^{\circ} \mathrm{C}$.

- Critical step: Any time aggregates are being pipetted, be sure to use wide orifice pipette tips to prevent any shearing of the EBs. This will also be important throughout the analysis stage.

vii. As an alternative to step $2 \mathrm{Av}$, use a p20 pipette set to $10 \mu \mathrm{L}$ to pick 10-15 EBs from the $10 \mathrm{~cm}$ dish and plate them onto a gelatin coated 24-well plate for a high-throughput way to test multiple conditions. Add $2 \mathrm{~mL}$ of the supplemented DFK5 described in step 2Aiv to each well.

viii. On day 4, prepare the differentiation media consisting of 10 mL DFK5 supplemented with $10 \mathrm{nM} \mathrm{RA}, 1 \mu \mathrm{M}$ pur and $5 \mu \mathrm{M}$ DAPT. In a $1.5 \mathrm{~mL}$ centrifuge tube, dilute $1 \mu \mathrm{L}$ of $20 \mathrm{mM}$ stock RA into $999 \mu \mathrm{L}$ of DFK5 to make a 1:1000 working dilution. In a separate $0.5 \mathrm{~mL}$ centrifuge tube, dilute $1 \mu \mathrm{L}$ of $50 \mathrm{mM}$ stock pur into $99 \mu \mathrm{L}$ of DFK5 to make a 1:100 working dilution. To $10 \mathrm{~mL}$ DFK5, add $5 \mu \mathrm{L}$ 1:1000 $20 \mathrm{mM}$ RA dilution, $20 \mu \mathrm{L}$ 1:100 $50 \mathrm{mM}$ pur dilution, and $5 \mu \mathrm{L}$ $10 \mathrm{mM}$ DAPT.

ix. Repeat steps $2 \mathrm{~A}(\mathrm{v})$ to $2 \mathrm{~A}(\mathrm{vi})$ to change the media. Incubate the culture dish at $37^{\circ} \mathrm{C}$. To change the media on the plated aggregates, aspirate the old media and add $2 \mathrm{~mL}$ fresh supplemented DFK5.

x. On day 6 of the differentiation, you can choose to analyze the differentiation efficiency using flow cytometry, ICC, and/or qPCR (Step 5) or continue with experimentation by dissociating the aggregates for enrichment or maturation as described in the next step.

xi. To dissociate, collect the media plus aggregates from the agar plate using a $10 \mathrm{~mL}$ stripette and dispense into a $15 \mathrm{~mL}$ conical tube. Allow the aggregates to settle to the bottom for 5-10 minutes. Resuspend in $1.5 \mathrm{~mL}$ warmed $0.25 \%$ TrypsinEDTA and incubate at $37^{\circ} \mathrm{C}$ for 10 minutes with intermittent trituration. Quench with Complete Medium containing $0.001 \%$ DNAse and count the cells.

B. Human V2a IN differentiation - TIMING: 17 days 
i. Culture hPSCs on Matrigel-coated T25 flasks to $70 \%$ confluence in mTeSR medium.

ii. Passage the cells as described in 1B.

iii. Calculate the number of cells needed to seed cells at approximately $50 \mathrm{k}$ cells per $\mathrm{cm}^{2}$. To differentiate 12 wells of a 24-well plate, calculate cell numbers based on 13 wells to account for approximately $10 \%$ pipette error; therefore, 1.3 million cells are needed. Add the cell suspension to a new 15 $\mathrm{mL}$ conical. To seed, $1 \mathrm{~mL}$ of media is added to each well. Therefore, to differentiate 12 wells, prepare $13 \mathrm{~mL}$ to account for approximately $10 \%$ pipette error. Add a volume of mTeSR $+10 \mu \mathrm{M} \mathrm{Y}-27632$ equal to $13 \mathrm{~mL}$ minus the volume of cell suspension. Add $0.26 \mu \mathrm{L}$ of LDN 193189 (final concentration of $0.2 \mu \mathrm{M}$ ) and $13 \mu \mathrm{L}$ of SB 435142 (final concentration of 10 $\mu \mathrm{M})$. Add $1 \mathrm{~mL}$ of the cell suspension supplemented with small molecules to Matrigel-coated wells. This corresponds to day 0 of the differentiation.

- Critical Step: The initial seeding density is critical to the success of the differentiation. Due to the difference in growth rates between hPSC lines, it is important to test a range of seeding densities to identify the optimal seeding density for your line (Fig. 9).

iv. On day 1 of the differentiation, prepare mTeSR containing 10 $\mu \mathrm{M}$ Y-27632, $10 \mu \mathrm{M}$ SB 435142 and $0.2 \mu \mathrm{M}$ LDN 193189. Add $2 \mathrm{~mL}$ media per well from this point forward (for 12 wells, prepare $25 \mathrm{~mL}$ of media to account for pipetting error).

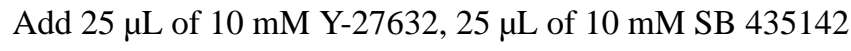
and $0.5 \mu \mathrm{L}$ of $10 \mathrm{mM}$ LDN 193189. Fully aspirate the media from the 24-well plate and add $2 \mathrm{~mL}$ of media to each well.

v. On day 3 of the differentiation, prepare mTeSR containing 10 $\mu \mathrm{M}$ SB 435142 and $0.2 \mu \mathrm{M}$ LDN 193189. To $25 \mathrm{~mL}$ of mTeSR, add $25 \mu \mathrm{L}$ of $10 \mathrm{mM}$ SB 435142 and $0.5 \mu \mathrm{L}$ of 10 mM LDN 193189. Fully aspirate the media from the 24-well plate and add $2 \mathrm{~mL}$ of media to each well.

vi. On day 5 of the differentiation, prepare NIM containing 10 $\mu \mathrm{M}$ SB 435142, $0.2 \mu \mathrm{M}$ LDN 193189, $0.4 \mu \mathrm{g} / \mathrm{mL}$ AA, 10 $\mathrm{ng} / \mathrm{mL}$ BDNF, and $100 \mathrm{nM} \mathrm{RA}$. In a $0.5 \mathrm{~mL}$ centrifuge tube, dilute $1 \mu \mathrm{L}$ of RA into $9 \mu \mathrm{L}$ of DMSO to make a 1:10 working dilution. To $25 \mathrm{~mL}$ of NIM, add $25 \mu \mathrm{L}$ of $10 \mathrm{mM} \mathrm{SB} 435142$, $0.5 \mu \mathrm{L}$ of $10 \mathrm{mM}$ LDN $193189,2 \mu \mathrm{L}$ of $5 \mathrm{mg} / \mathrm{mL}$ AA, $10 \mu \mathrm{L}$ of $25 \mu \mathrm{g} / \mathrm{mL}$ BDNF, and $1.25 \mu \mathrm{L}$ of 1:10 dilution of $20 \mathrm{mM}$ 
RA. Fully aspirate the media from the 24-well plate and add 2 $\mathrm{mL}$ of media to each well.

- Critical Step: It is important for the 1:10 small molecule dilutions to use DMSO to ensure that they fully go into solution. Do not freeze/thaw the dilutions; make a fresh dilution from the frozen stock before adding it to the solution for each media change.

vii. On day 7 of the differentiation, prepare NIM containing 0.4 $\mu \mathrm{g} / \mathrm{mL}$ AA, $10 \mathrm{ng} / \mathrm{mL}$ BDNF, $100 \mathrm{nM}$ RA, $100 \mathrm{nM}$ pur and 1 $\mu \mathrm{M}$ DAPT. In a $0.5 \mathrm{~mL}$ centrifuge tube, dilute $1 \mu \mathrm{L}$ of RA into $9 \mu \mathrm{L}$ of DMSO to make a 1:10 working dilution. In a separate $0.5 \mathrm{~mL}$ centrifuge tube, dilute $1 \mu \mathrm{L}$ of pur into $9 \mu \mathrm{L}$ of DMSO to make a 1:10 working dilution. To $25 \mathrm{~mL}$ of NIM, add $2 \mu \mathrm{L}$ of $5 \mathrm{mg} / \mathrm{mL}$ AA, $10 \mu \mathrm{L}$ of $25 \mu \mathrm{g} / \mathrm{mL}$ BDNF, $1.25 \mu \mathrm{L}$ of $1: 10$ dilution of $20 \mathrm{mM}$ RA, $0.5 \mu \mathrm{L}$ of 1:10 dilution of $50 \mathrm{mM}$ pur and $2.5 \mu \mathrm{L}$ of $10 \mathrm{mM}$ DAPT. Fully aspirate the media from the 24-well plate and add $2 \mathrm{~mL}$ of media to each well. Repeat this same feeding process on day 10 , day 13 and day 15 of the differentiation.

- Critical Step: Occasionally after multiple freeze/ thaws cycles, the pur stock can precipitate out of solution. If this happens, use a fresh aliquot.

?Troubleshooting: 2B(vii)

viii. On day 17 , the culture can be replated to enrich the V2a interneuron population (step 3), replated for physiological maturation (step 4), prepared for phenotypic analyses (step 5), or cryopreserved (Box 2).

ix. To dissociate the V2a interneuron cultures, aspirate old media from each well and wash once with $1 \mathrm{~mL}$ of PBS. Add $1 \mathrm{~mL}$ of Accutase to each well and incubate at $37^{\circ} \mathrm{C}$ for 15 minutes. Triturate approximately 5 times with a p1000 (not a wideorifice tip) to break up cell layers and return to the incubator for 15 minutes. Repeat trituration and incubate for 15 more minutes for a total incubation of 45 minutes.

x. Transfer the dissociated cell suspension to a fresh $15 \mathrm{~mL}$ conical tube and dilute with a volume of PBS equal to 3 times the volume of Accutase. Count the cells. Centrifuge at $200 \mathrm{xg}$ for 5 minutes at room temperature. 


\section{Enrichment of V2a Interneurons - TIMING: $\mathbf{1}$ day for mouse, $\mathbf{3}$ days for human}

3) If you are using the Chx10-Puro mESC and wish to enrich mouse V2a interneurons, follow option A. If you wish to enrich human V2a interneurons, follow option B. Otherwise proceed to the next step.

A. Enrichment of mouse V2a interneurons using antibiotic selection with the transgenic Chx 10-Puro mESC line - TIMING: 1 day

i. On day 6 of the differentiation, dissociate and count differentiated neurons as described in step $2 \mathrm{~A}(\mathrm{x})$. Centrifuge at $300 \mathrm{x}$ g for 5 minutes at room temperature. Resuspend cells in the selection medium and seed at $5 \times 10^{6}$ cells per $\mathrm{cm}^{2}$ onto laminin coated plates.

CRITICAL STEP Whilst the cells are dissociating, proceed with the next step.

?Troubleshooting: 3A(i)

ii. While the cells are dissociating, prepare the selection medium. Selection medium consists of DFK5NB supplemented with B27, 100x GlutaMAX, $10 \mathrm{ng} / \mathrm{mL}$ NT-3, $10 \mathrm{ng} / \mathrm{mL}$ GDNF, $10 \mathrm{ng} / \mathrm{mL}$ BDNF and $2 \mu \mathrm{g} / \mathrm{mL}$ puromycin. For example, to select in a T-25 flask, prepare $5 \mathrm{~mL}$ DFK5NB containing $100 \mu \mathrm{L} \mathrm{B} 27,50 \mu \mathrm{L}$ GlutaMAX, and $1 \mu \mathrm{L} 10$ $\mathrm{mg} / \mathrm{mL}$ puromycin stock.

- Critical step: Note that the concentration of puromycin and cell density will control the degree of selection. At $4 \mu \mathrm{g} / \mathrm{mL}$ puromycin, there will be more cell death, but a more enriched population than at 2 $\mu \mathrm{g} / \mathrm{mL}$ puromycin.

iii. After 24 hours in the selection medium cells can be matured by, replating at a different density (step 4A), aggregating (Box 1) or directly maturing at the current density. To mature at current density, replace the selection medium with DFK5NB containing B27, 100x GlutaMAX, $10 \mathrm{ng} / \mathrm{mL}$ NT-3, $10 \mathrm{ng} / \mathrm{mL}$ GDNF and $10 \mathrm{ng} / \mathrm{mL}$ BDNF. Replace the media every 2 days throughout the maturation process.

B. Enrichment of human V2a interneurons by replating - TIMING: 3 days

i. Dissociate the human V2a interneuron cultures as described in step 2B(ix-x). If replating multiple wells, combine wells during the collection step. For example, to replate 6 wells, combine 6 wells of dissociated cells in a $50 \mathrm{~mL}$ conical tube and dilute the $6 \mathrm{~mL}$ of Accutase with $18 \mathrm{~mL}$ PBS. 
CRITICAL STEP Proceed with the next step whilst the cells are dissociating.

ii. While the cells are dissociating, prepare $2 \mathrm{~mL}$ per well of the same medium used on day 15 and supplement with $1 \mu \mathrm{M}$ Y-27632. For 6 wells, take $13 \mathrm{~mL}$ NIM and add $1.04 \mu \mathrm{L}$ of 5 $\mathrm{mg} / \mathrm{mL}$ AA, $5.2 \mu \mathrm{L}$ of $25 \mu \mathrm{g} / \mathrm{mL}$ BDNF, $0.65 \mu \mathrm{L}$ of 1:10 dilution of $20 \mathrm{mM}$ RA, $0.26 \mu \mathrm{L}$ of 1:10 dilution of $50 \mathrm{mM}$ pur, $1.3 \mu \mathrm{L}$ of $10 \mathrm{mM}$ DAPT, and $1.3 \mu \mathrm{L}$ of $10 \mathrm{mM}$ Y-27632.

- $\quad$ Critical step: Note that a 10-fold reduction in concentration of Y-27632 (1 $\mu \mathrm{M}$ Y-27632 compared to $10 \mu \mathrm{M}$ ) is used in this step. It is normal to observe an increased amount of cell death.

iii. Aspirate supernatant from the conical tube of dissociated cells and resuspend cell pellet with $12 \mathrm{~mL}$ of the prepared NIM medium. Plate onto a fresh Matrigel-coated 24-well plate with $2 \mathrm{~mL}$ of cell suspension per well. Return to the incubator for 3 days before analysis.

\section{Physiological maturation - TIMING: Variable}

4) Following the V2a induction or enrichment process, the cells can be cultured for long periods of time (up to 40+ days for mouse and 100+ days for human) until they express important electrophysiological markers of V2a interneurons such as VGLUT2 (by day 21 for mouse and 60 for human). To mature mouse cultures, follow option A to use monolayer culture with or without selection.

Alternatively, functional maturation of mouse V2a interneurons can be improved with co-culture with glial cells. Such co-culture can be carried out by dissociating selected cells and reseeding with glial cells at the desired ratio, or with the cells as aggregates, as described in Box 1. To mature human cells follow option C.

A. Maturation of mouse cultures in monolayer-TIMING: variable

i. To mature the cells in monolayer without selection, dissociate aggregates as described in step $2 \mathrm{~A}(\mathrm{x})$. Replate at a concentration of 200k-500k cells per $\mathrm{cm}^{2}$ in DFK5NB medium containing B27, 100x GlutaMAX, 10 ng/mL NT-3, 10 ng/mL GDNF and 10 ng/mL BDNF.

ii. Replace half of the medium every 2 days throughout the maturation process. Move to phenotypic analyses at the desired time point.

B. Maturation of human cultures - TIMING: variable

i. Dissociate and count cultures as described in step 2B(ix-x).

ii. Seed cells onto a Matrigel-coated surface at a concentration of $200 \mathrm{k}$ cells per $\mathrm{cm}^{2}$ with $500 \mu \mathrm{L}$ of the same medium as described in step 2B(vii) supplemented with $10 \mu \mathrm{M}$ Y-27632. To mature for patch clamp 
analysis, plate onto a 48-well plate and transfer to glass coverslips before patch clamp. To perform high quality imaging (both immunostaining and calcium imaging), plate onto a $\mu$-slide.

iii. Three days after seeding, prepare BrainPhys medium supplemented with SM1 and $10 \mathrm{ng} / \mathrm{mL}$ of BDNF, GDNF, CNTF and IGF. In a 48-well plate, carefully remove $250 \mu \mathrm{L}$ of the old medium and replace with 500 $\mu \mathrm{L}$ of fresh medium for an approximate volume of $750 \mu \mathrm{L}$. Every 4 to 5 days, change the media by removing $500 \mu \mathrm{L}$ old medium and replacing with $500 \mu \mathrm{L}$ fresh medium. When culturing in a $\mu$-slide, the maximum working volume is $250 \mu \mathrm{L}$. Remove and replace $150 \mu \mathrm{L}$ every 3 days. Move to phenotypic analyses at desired time point.

?Troubleshooting: 4B(iii)

\section{Phenotypic analyses - TIMING: variable}

At the end of the differentiation, selection and maturation processes, differentiation efficiency can be assessed by flow cytometry (A), immunostaining (B) and qPCR (C). Alternatively, calcium imaging can be carried out (D and E).

A. Flow cytometry - TIMING: 1 day

i. Dissociate and count cell cultures as described in step $2 \mathrm{~A}(\mathrm{x})$ for mouse and $2 \mathrm{~B}(\mathrm{xi}-\mathrm{x})$ for human.

ii. Resuspend dissociated cells in $500 \mu \mathrm{L}$ fix/perm buffer and incubate at $4^{\circ} \mathrm{C}$ for 40 minutes.

- $\quad$ Critical step: Proceed with the rest of the staining and analysis on the same day. Storing the samples over night may result in inaccurate results.

iii. Add $500 \mu \mathrm{L}$ perm/wash buffer to each sample and centrifuge at $200 \mathrm{x} \mathrm{g}$ for 5 minutes at room temperature.

iv. To block non-specific binding, resuspend the cell pellet in each sample with $500 \mu \mathrm{L}$ perm/wash buffer containing $5 \%$ normal donkey serum (NDS). Incubate at $4^{\circ} \mathrm{C}$ for 15 minutes.

v. Centrifuge at $200 \mathrm{x}$ g for 5 minutes at room temperature. Resuspend cell pellet in $200 \mu \mathrm{L}$ of perm/wash buffer containing $2 \%$ NDS. For each sample, pipette $90 \mu \mathrm{L}$ of the cell suspension into 2 wells of a round bottom 96-well plate. One well will be stained with CHX10 and the other well will be stained with an IgG control. Prepare a 1:100 dilution of the CHX10 and mouse IgG antibody with $1 \mu \mathrm{L}$ antibody and $99 \mu \mathrm{L}$ mouse IgG. Pipette $9 \mu \mathrm{L}$ of the 1:100 CHX10 dilution into the wells being stained with $\mathrm{CHX} 10$ and $3.6 \mu \mathrm{L}$ of the $1: 100 \mathrm{IgG}$ dilution into the control wells. Incubate at $4^{\circ} \mathrm{C}$ for 45 minutes. 
vi. Centrifuge the 96 -well plate at $800 \mathrm{x}$ g for 3 minutes. Remove supernatant by quickly flicking the plate upside down over a sink. Wash by adding $100 \mu \mathrm{L}$ perm/wash buffer to each well. Centrifuge the 96well plate at $800 \mathrm{x}$ g for 3 minutes. Repeat the wash step.

vii. Prepare secondary solution containing perm/wash buffer with $2 \%$ NDS and donkey anti mouse 488 alexa fluor secondary at a concentration of 1:200. Add $100 \mu \mathrm{L}$ of secondary solution to each well. Be sure to pipette up and down to resuspend cells. Incubate at $4^{\circ} \mathrm{C}$ for 45 minutes.

viii. Repeat wash step $5 \mathrm{~A}(\mathrm{vi})$

ix. Resuspend samples with $300 \mu \mathrm{L}$ PBS and pass through filtered flow cytometry tubes. Centrifuge at $800 \mathrm{x}$ g for 30 seconds to pull cell suspension through the filter. Vortex to resuspend the cells and run analysis on a BD accuri flow cytometer or a similar instrument. Figure 8 provides an example of expected scatter plots for $\mathrm{CHX} 10$ and IgG staining.

?Troubleshooting: 5A(ix)

B. Immunocytochemistry (ICC) - TIMING: 3 days

CRITICAL Here we describe the volumes necessary to perform ICC in a 24-well plate. Volumes can be scaled down for staining in a 48 -well plate or a $\mu$-slide.

i. To perform ICC, wash one time with $1 \mathrm{~mL}$ PBS. Fix the cell layers in a fume hood with $500 \mu \mathrm{L}$ per well of $4 \%$ paraformaldehyde for 30 minutes at room temperature.

?Troubleshooting: 5B(i)

ii. Remove and safely dispose of paraformaldehyde. Wash 1 time with $1 \mathrm{~mL}$ PBS per well.

- $\quad$ Pause point: you can fill each well with $2 \mathrm{~mL}$ PBS, wrap the plate with parafilm and store it at $4^{\circ} \mathrm{C}$ for 2 weeks before staining.

iii. Permeabilize the cells by adding $500 \mu \mathrm{L}$ of $0.1 \%$ Triton $\mathrm{X}$ in PBS and incubate at $4^{\circ} \mathrm{C}$ for 15 minutes.

iv. Block for non-specific binding by adding $500 \mu \mathrm{L}$ PBS containing 5\% NDS to each well. Incubate at $4^{\circ} \mathrm{C}$ for 1 hour.

v. Calculate the volume of antibody solution needed for $250 \mu \mathrm{L}$ in each well. Prepare PBS with 2\% NDS and add the proper antibody concentrations as listed in Table 1. Incubate at $4^{\circ} \mathrm{C}$ overnight.

vi. Wash three times with $1 \mathrm{~mL}$ PBS for 15 minutes each at room temperature.

vii. Calculate the volume of antibody solution you will need for $250 \mu \mathrm{L}$ per well.

Prepare PBS with 2\% NDS and add corresponding secondaries at a concentration of 1:200. Incubate at $4^{\circ} \mathrm{C}$ overnight. 
?Troubleshooting: 5B(vii)

viii. Wash 3 times with $1 \mathrm{~mL}$ PBS for 15 minutes each at room temperature.

ix. Add $500 \mu \mathrm{L}$ of PBS with 1:1000 Hoechst to each well and incubate at room temperature for 10 minutes.

x. Wash 1 time with $1 \mathrm{~mL}$ PBS for 15 minutes each at room temperature.

xi. Add $300 \mu \mathrm{L}$ PBS to each well and image on a fluorescent inverted microscope.

xii. When imaging is complete, add $2 \mathrm{~mL}$ PBS and wrap the plate with parafilm and store at $4^{\circ} \mathrm{C}$ for up to 1 month.

C. Quantitative polymerase chain reaction (qPCR) - TIMING: variable

i. To lyse the cells for PCR analysis, wash the sample 1 time with PBS and add $700 \mu \mathrm{L}$ of lysis buffer. Pipette up and down to fully lyse the cells.

- $\quad$ Pause Point: Lysed samples can be stored at $-80^{\circ} \mathrm{C}$ for at least a year.

ii. Follow manufacturer's instructions to isolate RNA using Qiagen's RNeasy kit.

iii. Measure the concentration and quality of RNA using a nanodrop.

- $\quad$ Pause Point: RNA can be stored at $-80^{\circ} \mathrm{C}$ for up to a year

iv. Convert the RNA to cDNA using the iScript ${ }^{\mathrm{TM}} \mathrm{cDNA}$ synthesis kit.

- $\quad$ Pause Point: cDNA can be stored at $-20^{\circ} \mathrm{C}$ for up to a year.

v. Prepare the qPCR reaction mix according to the master mix instructions. A list of primers used to characterize the V2a interneuron population can be found in Table 2 .

D. Calcium analysis of mouse cells - TIMING: variable

i. For calcium imaging, seed co-cultured aggregates onto laminin-coated wells in DFK5NB medium supplemented with B27, 100x GlutaMAX, $5 \mathrm{ng} / \mathrm{mL}$ of NT-3, GDNF, BDNF, PDGF, and $0.5 \mu \mathrm{L} / \mathrm{mL}$ of reconstituted AAV1-hSyn-GCaMP6f vector.

ii. After 24 hours, replace the medium to DFK5NB supplemented with growth factors and change the media every 3 days.

iii. After a week of culture, change to Neurobasal medium supplemented with growth factors. Change media every 3 days for an additional week. We image calcium transients on an inverted microscope with a ORCA-R2 camera at a rate of 4 frames per second.

E. Calcium analysis of human cells - TIMING: variable 
i. If an endogenous calcium sensor is not available, treat the cells with the calcium sensitive dye, Fluo-4 AM. To do this, dilute the 5 mM Fluo- 4 AM dye to $5 \mu \mathrm{M}$ into BrainPhys medium with no supplements. For one well of a 48-well plate, add $250 \mu \mathrm{L}$ of Brainphys with Fluo-4 AM. Incubate for 30 minutes at $37^{\circ} \mathrm{C}$.

ii. Aspirate dye solution and wash well 1 time with $250 \mu \mathrm{L}$ PBS. Recover the cells in $250 \mu \mathrm{L}$ BrainPhys supplemented with SM1 and $10 \mathrm{ng} / \mathrm{mL}$ of BDNF, GDNF, CNTF and IGF for 30 minutes at $37^{\circ} \mathrm{C}$. The cells are now ready to be imaged.

iii. We perform imaging using the Zeiss Axio Observer inverted microscope with a Hamamatsu orca flash 4.0 camera at an exposure time of $70 \mathrm{~ms}$. The montage images and video shown in Fig. 6d and Video 2 were performed at 45 days of culture.

\section{TIMING}

Step 1A, Mouse pluripotent culture

Step 1B, Human pluripotent culture

Step 2A, Mouse V2a interneuron differentiation: $6 \mathrm{~d}$

Step 2B, Human V2a interneuron differentiation: $17 \mathrm{~d}$

Step 3A, Mouse V2a interneuron enrichment: $1 \mathrm{~d}$

Step 3B, Human V2a interneuron enrichment: $3 \mathrm{~d}$

Step 4A, Maturation of mouse cultures: variable

Step 4B, Maturation of human cultures: variable

Step 5A, Flow cytometry: $1 \mathrm{~d}$

Step 5B, Immunocytochemistry: $3 \mathrm{~d}$

Step 5C, PCR: variable

Step 5D, Calcium analysis of moues cells: variable

Step 5E, Calcium analysis of human cells: variable

Box 1, Mouse V2a interneuron and pMN aggregate formation: $2 \mathrm{~d}$

Box 2, Human V2a interneuron cryopreservation and thaw: $3 \mathrm{~d}$

\section{?TROUBLESHOOTING}

For troubleshooting guidance see Table 3. 


\section{ANTICIPATED RESULTS}

When performing the differentiation with mouse cells, the expected CHX10 percentages will be approximately $20 \%$ at day 6 (Fig. $4 \mathrm{a}$ ). The cultures will have increased levels of V2a gene transcripts including $C h \times 10$ and $L h x 3$ (Fig. 4e). Following antibiotic selection, the CHX10 percentage can be enriched to at least $75 \%$ (Fig. 5a). The process of selection can hinder the maturation and survivability of the V2a cultures; however, purified Olig2 expressing cells can be added to the cultures to improve survival following antibiotic selection (Fig. 10). Mouse V2a interneurons express minimally detectable amounts of VGlut2 at 2 weeks of maturation but by 4 weeks, the V2a neurons have robust VGlut2 expression and extensive neurite growth (Fig. 6a). When mixed with Olig2 expressing cells, the V2a cultures display spontaneous calcium flux activity indicative of maturation (Fig. 6c).

When performing the differentiation with human cells, CHX10 percentages can range from 20 to $50 \%$ depending on the PSC line (Fig. $4 \mathrm{~b}$ ). Gene expression analysis reveals upregulation of committed V2a interneuron markers, including $C H X 10$ and FOX14, while markers for adjacent neuronal domains ( $H B 9$ ) and retinal cells $(C R X)$ should be low (Fig. 4f). The V2a interneuron population can be enriched as much as 6-fold by dissociating and replating the cells with $1 \mu \mathrm{M}$ Y-27632. However, the largest enrichment from replating is observed with lower initial differentiation efficiencies (Fig. 5b). As the V2a cultures mature, the cultures should express VGlut2 by day 60 (Fig. 6b). The V2a cultures expressing GCaMP (or labeled with a fluorescent dye) display spontaneous calcium transients by day 45 (Fig. 6d). Lastly, the human V2a interneuron cultures can be cyropreserved without adversely affecting CHX10 percentage and can be used for studies at a later date (Fig. 7).

The V2a differentiation has been successfully performed with all of the mESC and hPSC lines examined to date. However we have found that the success of different hPSC cell lines is very sensitive to the initial seeding density (Figure 9). When attempting a differentiation for the first time, it is important to test several seeding densities ( $5 \mathrm{k}$ to $100 \mathrm{k}$ cells $/ \mathrm{cm}^{2}$ ). It is suggested to determine which seeding density forms a confluent monolayer by day 3 of the differentiation and continues to yield a dense layer of neurons at the end of the differentiation.

Together, this protocol describes how to differentiate, mature, and characterize V2a interneurons from mouse and human PSCs. A stem cell-derived population of V2a interneurons can be used to elucidate their phenotypic and electrophysiological properties as well as their regenerative potential following spinal cord injuries and degenerative diseases, such as ALS.

\section{Supplementary Material}

Refer to Web version on PubMed Central for supplementary material.

\section{ACKNOWLEDGEMENTS}

We would like to acknowledge Dr. Chelsea Brown for her dedication and hard work in co-discovering the original mouse V2a interneuron protocol. In addition, we acknowledge the Gladstone Stem Cell Core for providing cell culture facilities and the Gladstone Communications department for graphic creation. This work was made possible 
through funding by NIH NINDS F31 NS090760 (N.R.I), CIRM LA1 C14-08015 (T.C.M), The Roddenberry Foundation L02593 (T.C.M), and NIH NINDS R01 NS090617 (S.S.E).

\section{REFERENCES}

1. Crone SA et al. Irregular Breathing in Mice following Genetic Ablation of V2a Neurons. J Neurosci 32, 7895-7906, doi:10.1523/JNEUROSCI.0445-12.2012 (2012). [PubMed: 22674265]

2. Smith JC, Ellenberger HH, Ballanyi K, Richter DW \& Feldman JL Pre-Botzinger complex: a brainstem region that may generate respiratory rhythm in mammals. Science 254, 726-729 (1991). [PubMed: 1683005]

3. Crone SA et al. Genetic ablation of V2a ipsilateral interneurons disrupts left-right locomotor coordination in mammalian spinal cord. Neuron 60, 70-83, doi:10.1016/j.neuron.2008.08.009 (2008). [PubMed: 18940589]

4. Al-Mosawie A, Wilson JM \& Brownstone RM Heterogeneity of V2-derived interneurons in the adult mouse spinal cord. Eur J Neurosci 26, 3003-3015, doi:10.1111/j.1460-9568.2007.05907.x (2007). [PubMed: 18028108]

5. Romer SH et al. Accessory respiratory muscles enhance ventilation in ALS model mice and are activated by excitatory V2a neurons. Exp Neurol 287, 192-204, doi:10.1016/ j.expneurol.2016.05.033 (2017). [PubMed: 27456268]

6 . Bareyre FM et al. The injured spinal cord spontaneously forms a new intraspinal circuit in adult rats. Nat Neurosci 7, 269-277, doi:10.1038/nn1195 (2004). [PubMed: 14966523]

7. Zholudeva LV et al. Transplantation of Neural Progenitors and V2a Interneurons after Spinal Cord Injury. J Neurotrauma, doi:10.1089/neu.2017.5439 (2018).

8. Brown CR, Butts JC, McCreedy DA \& Sakiyama-Elbert SE Generation of v2a interneurons from mouse embryonic stem cells. Stem Cells Dev 23, 1765-1776, doi:10.1089/scd.2013.0628 (2014). [PubMed: 24650073]

9. Iyer NR, Huettner JE, Butts JC, Brown CR \& Sakiyama-Elbert SE Generation of highly enriched V2a interneurons from mouse embryonic stem cells. Exp Neurol 277, 305-316, doi:10.1016/ j.expneurol.2016.01.011 (2016). [PubMed: 26784005]

10. Butts JC et al. Differentiation of V2a interneurons from human pluripotent stem cells. Proc Natl Acad Sci U S A 114, 4969-4974, doi:10.1073/pnas.1608254114 (2017). [PubMed: 28438991]

11. Marklund U et al. Detailed expression analysis of regulatory genes in the early developing human neural tube. Stem Cells Dev 23, 5-15, doi:10.1089/scd.2013.0309 (2014). [PubMed: 24007338]

12. Philippidou P \& Dasen JS Hox genes: choreographers in neural development, architects of circuit organization. Neuron 80, 12-34, doi:10.1016/j.neuron.2013.09.020 (2013). [PubMed: 24094100]

13. Okada Y, Shimazaki T, Sobue G \& Okano H. Retinoic-acid-concentration-dependent acquisition of neural cell identity during in vitro differentiation of mouse embryonic stem cells. Dev Biol 275, 124-142, doi:10.1016/j.ydbio.2004.07.038 (2004). [PubMed: 15464577]

14. Patten I \& Placzek M. The role of Sonic hedgehog in neural tube patterning. Cell Mol Life Sci 57, 1695-1708, doi:10.1007/PL00000652 (2000). [PubMed: 11130176]

15. Imayoshi I et al. Oscillatory control of factors determining multipotency and fate in mouse neural progenitors. Science 342, 1203-1208, doi:10.1126/science.1242366 (2013). [PubMed: 24179156]

16. Imayoshi I, Sakamoto M, Yamaguchi M, Mori K \& Kageyama R. Essential roles of Notch signaling in maintenance of neural stem cells in developing and adult brains. J Neurosci 30, 34893498, doi:10.1523/JNEUROSCI.4987-09.2010 (2010). [PubMed: 20203209]

17. Shimojo H, Ohtsuka T \& Kageyama R. Oscillations in notch signaling regulate maintenance of neural progenitors. Neuron 58, 52-64, doi:10.1016/j.neuron.2008.02.014 (2008). [PubMed: 18400163]

18. Del Barrio MG et al. A regulatory network involving Foxn4, Mash1 and delta-like 4/Notch1 generates V2a and V2b spinal interneurons from a common progenitor pool. Development 134, 3427-3436, doi:10.1242/dev.005868 (2007). [PubMed: 17728344]

19. Amoroso MW et al. Accelerated high-yield generation of limb-innervating motor neurons from human stem cells. J Neurosci 33, 574-586, doi:10.1523/JNEUROSCI.0906-12.2013 (2013). [PubMed: 23303937] 
20. Wichterle H, Lieberam I, Porter JA \& Jessell TM Directed differentiation of embryonic stem cells into motor neurons. Cell 110, 385-397 (2002). [PubMed: 12176325]

21. Hoang PT et al. Subtype Diversification and Synaptic Specificity of Stem Cell-Derived Spinal Interneurons. Neuron 100, 135-149 e137, doi:10.1016/j.neuron.2018.09.016 (2018). [PubMed: 30308166]

22. Sternfeld MJ et al. Speed and segmentation control mechanisms characterized in rhythmicallyactive circuits created from spinal neurons produced from genetically-tagged embryonic stem cells. Elife 6, doi:10.7554/eLife.21540 (2017).

23. Xu H \& Sakiyama-Elbert SE Directed Differentiation of V3 Interneurons from Mouse Embryonic Stem Cells. Stem Cells Dev 24, 2723-2732, doi:10.1089/scd.2015.0122 (2015). [PubMed: 26165862]

24. White N \& Sakiyama-Elbert S. Derivation of specific neural populations from pluripotent cells for understanding and treatment of spinal cord injury. Dev Dyn, doi:10.1002/dvdy.24680 (2018).

25 . Dougherty KJ et al. Locomotor rhythm generation linked to the output of spinal shox 2 excitatory interneurons. Neuron 80, 920-933, doi:10.1016/j.neuron.2013.08.015 (2013). [PubMed: 24267650]

26. Panayi $\mathrm{H}$ et al. Sox 1 is required for the specification of a novel p2-derived interneuron subtype in the mouse ventral spinal cord. J Neurosci 30, 12274-12280, doi:10.1523/ JNEUROSCI.2402-10.2010 (2010).

27. Chambers SM et al. Highly efficient neural conversion of human ES and iPS cells by dual inhibition of SMAD signaling. Nat Biotechnol 27, 275-280, doi:10.1038/nbt.1529 (2009). [PubMed: 19252484]

28. Kreitzer FR et al. A robust method to derive functional neural crest cells from human pluripotent stem cells. Am J Stem Cells 2, 119-131 (2013). [PubMed: 23862100]

29. Mandegar MA et al. CRISPR Interference Efficiently Induces Specific and Reversible Gene Silencing in Human iPSCs. Cell Stem Cell 18, 541-553, doi:10.1016/j.stem.2016.01.022 (2016). [PubMed: 26971820]

30. McCreedy DA, Rieger CR, Gottlieb DI \& Sakiyama-Elbert SE Transgenic enrichment of mouse embryonic stem cell-derived progenitor motor neurons. Stem Cell Res 8, 368-378, doi:10.1016/ j.scr.2011.12.003 (2012). [PubMed: 22297157] 


\section{Box 1:}

\section{Aggregate co-culture of selected V2a interneurons with glial cells - TIMING: 2 days}

1. For aggregation of selected mouse V2a interneuron cultures, dissociate selected cells using Accutase at $37^{\circ} \mathrm{C}$ for 10 minutes and count cells as described in step $2 \mathrm{~A}(\mathrm{x})$. Obtain glial cells using the protocols described in McCreedy et $a \beta^{30}$.

2. While cells are dissociating, prepare AggreWell plate. Add $500 \mu \mathrm{L}$ AggreWell Rinsing Solution to each well of 24-well AggreWell Plate and centrifuge at maximum speed for 5 minutes. If any bubbles remain trapped, centrifuge for an additional 5 minutes to ensure that all bubbles have been removed from microwells.

3. Aspirate AggreWell Rinsing Solution from the wells and rinse wells twice with DMEM/F12. Add $500 \mu \mathrm{L}$ mouse maturation medium described in step $4 \mathrm{~A}(\mathrm{i})$ to each well. Centrifuge plate at maximum speed for 5 minutes to remove any bubbles from the wells.

4. Count 500,000 cells per AggreWell well to obtain aggregates $\sim 100-150 \mu \mathrm{m}$ in diameter. For a 1:1 ratio of V2a interneurons to glial cells, count 250,000 of each population and mix them gently. Centrifuge at $200 \mathrm{x} g$ for 5 minutes at room temperature to pellet cells.

5. Gently resuspend cells in $500 \mu \mathrm{L}$ mouse maturation medium described in step 4A(i). Pipette cell suspension into prepared AggreWell plate and triturate once to distribute the cells evenly. Centrifuge at $200 \mathrm{x}$ g for 5 minutes at room temperature.

6. Allow aggregates to form for 48 hours. Perform half-media changes daily, pipetting gently to avoid dislodging the aggregates. When formed, the aggregates will have a noticeable boundary (Fig. 10b). To collect aggregates for reseeding on laminin-coated wells (Fig. 10c) or for transplantation, pipette hard using a wide-orifice tip to dislodge the aggregates from the microwells. 


\section{Box 2:}

\section{Cryopreservation of human V2a interneuron cultures - TIMING: 3 days}

1. To freeze human V2a interneuron cultures, dissociate and freeze 124 -well per cryovial. Dissociate and count cultures as described in step 2B(ix-x).

2. While cells are dissociating, prepare the freezing medium and cryovials. You will need $1 \mathrm{~mL}$ of freezing medium per vial of cells. For the freezing medium, first prepare a mixture of $80 \%$ FBS with 20\% DMSO. Prepare NIM containing the factors as described in step $2 \mathrm{~B}$ (vii) and supplement with $10 \mu \mathrm{M}$ Y-27632. Mix the FBS mixture and NIM media in a 1:1 ratio to complete freezing medium preparation.

3. Similar to the replating in step $3 \mathrm{~B}$, wells can be combined at this step. Centrifuge at $200 \mathrm{x}$ g for 5 minutes at room temperature to pellet cells.

4. Resuspend the pellet with $1 \mathrm{ml}$ of freezing medium for every dissociated well, which should contain approximately 6 million cells for a normal differentiation. For example, after dissociating 6 wells, resuspend the pellet in $6 \mathrm{~mL}$ of freezing medium and dispense $1 \mathrm{~mL}$ in to each cryovial.

5. Place cyrovials into a Mr. Frosty and place in $-80^{\circ} \mathrm{C}$ for 24 hours. Move the vials to liquid nitrogen storage.

- $\quad$ Pause point: Vials can be stored in the liquid nitrogen for at least a year before being thawed.

6. To thaw cells, briefly open the cryovial in a biosafety cabinet to release any built up pressure. Incubate the cryovial in a $37^{\circ} \mathrm{C}$ water bath until $\sim 90 \%$ of ice crystals have dissolved.

?Troubleshooting

7. Dilute cell mixture into $4 \mathrm{~mL}$ NIM containing $5 \mu \mathrm{L} 10 \mathrm{mM} \mathrm{Y}-27632$. Centrifuge at $200 \mathrm{x} \mathrm{g}$ for 5 minutes at room temperature.

8. Prepare 1 Matrigel-coated well of a 24-well plate per cryovial to be thawed. Resuspend the cell pellet (approximately 2 million cells) with $2 \mathrm{~mL}$ NIM containing factors mentioned in step 2B(vii) supplemented with $10 \mu \mathrm{M}$ Y-27632 and dispense onto a Matrigel coated well of a 24-well plate. Incubate at $37^{\circ} \mathrm{C}$ and allow to recover for at least three days before use.

?Troubleshooting

END BOX 


\section{Key References}

1. Differentiation of V2a interneurons from human pluripotent stem cells. doi: 10.1073/pnas.1608254114.

2. Generation of highly enriched V2a interneurons from mouse embryonic stem cells. doi: 10.1016/j.expneurol.2016.01.011.

3. Generation of V2a interneurons from mouse embryonic stem cells. doi: 10.1089/scd.2013.0628. 


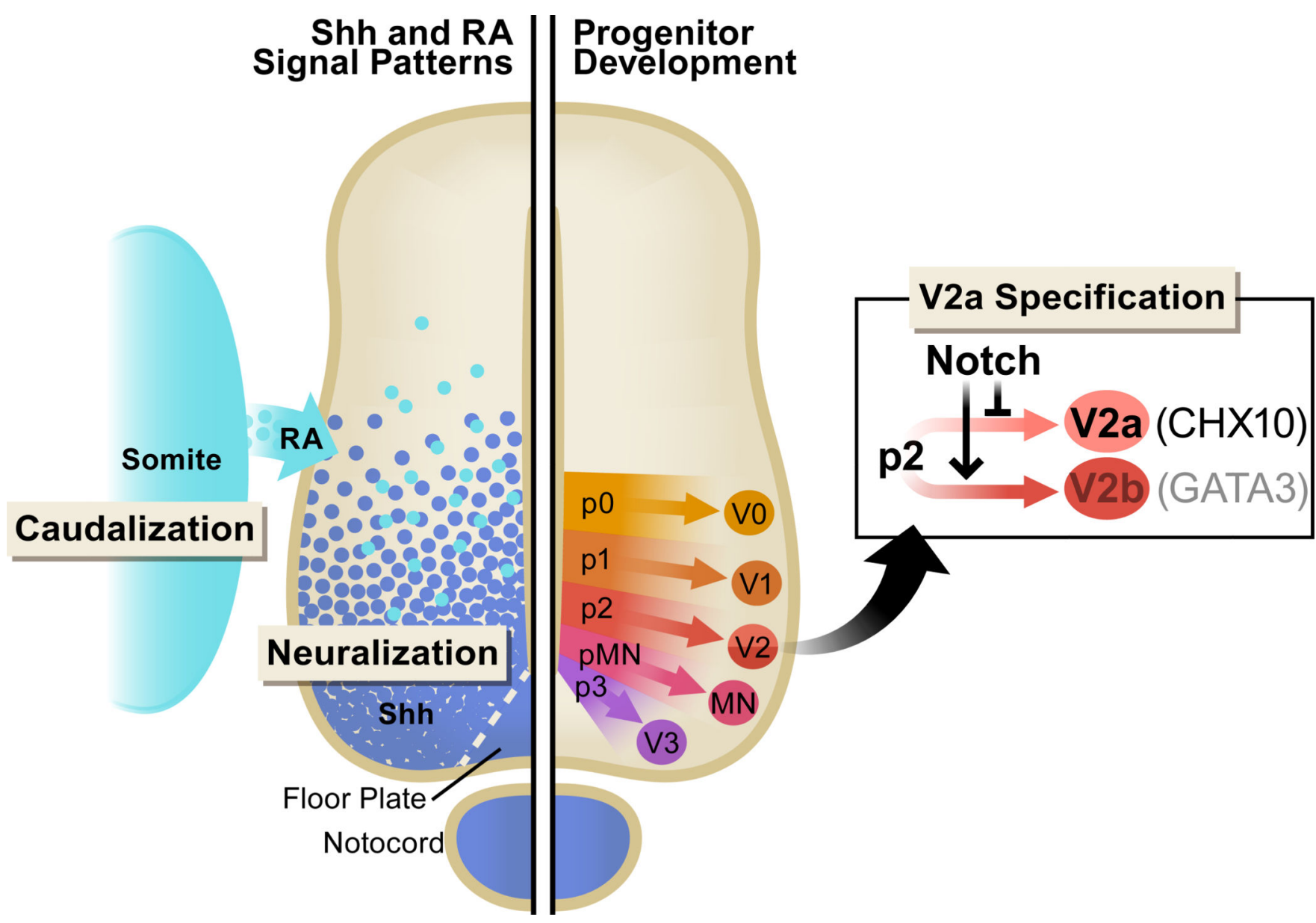

Figure 1 .

Schematic of developing neural tube. Retinoic acid (RA), Sonic Hedgehog (Shh) and Notch signaling have specific roles in the specification of interneuron progenitor domains ( $\mathrm{p} 3-$ p0). Adapted from Butts et al. ${ }^{10}$ 

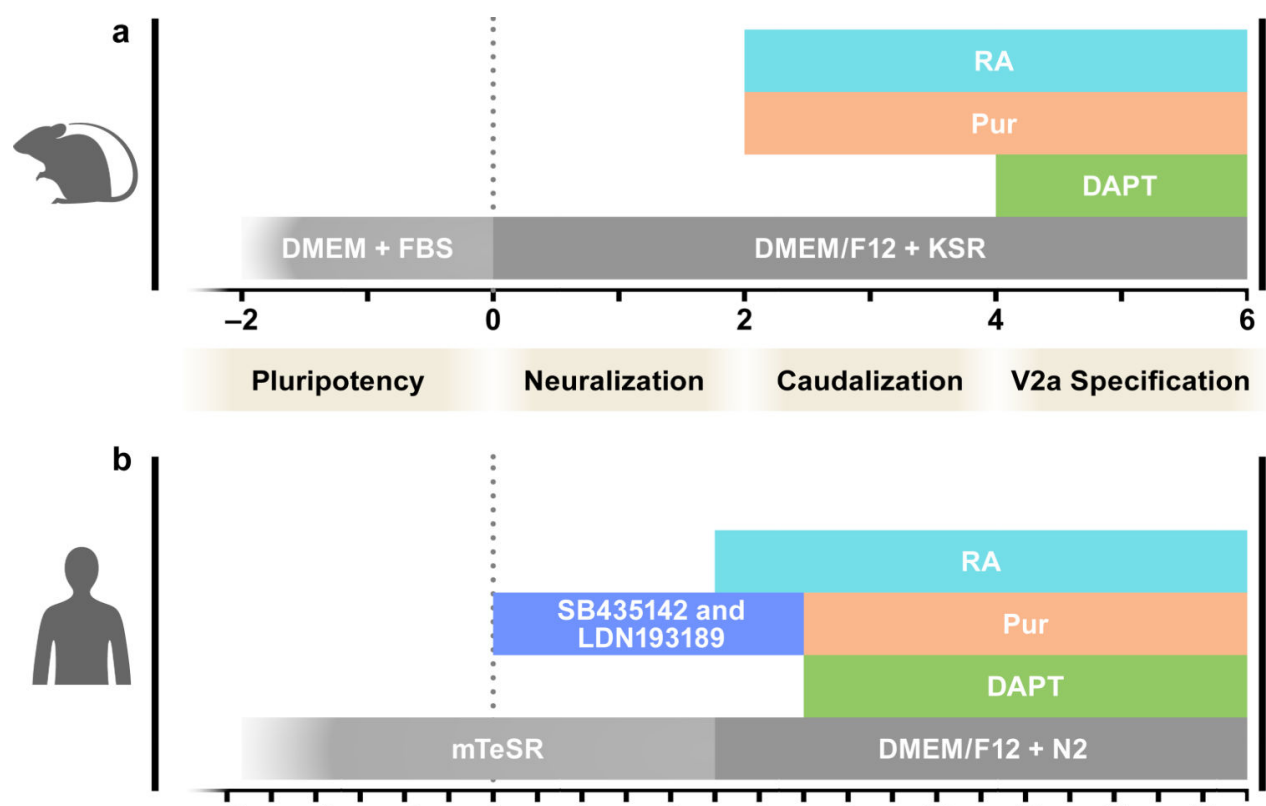

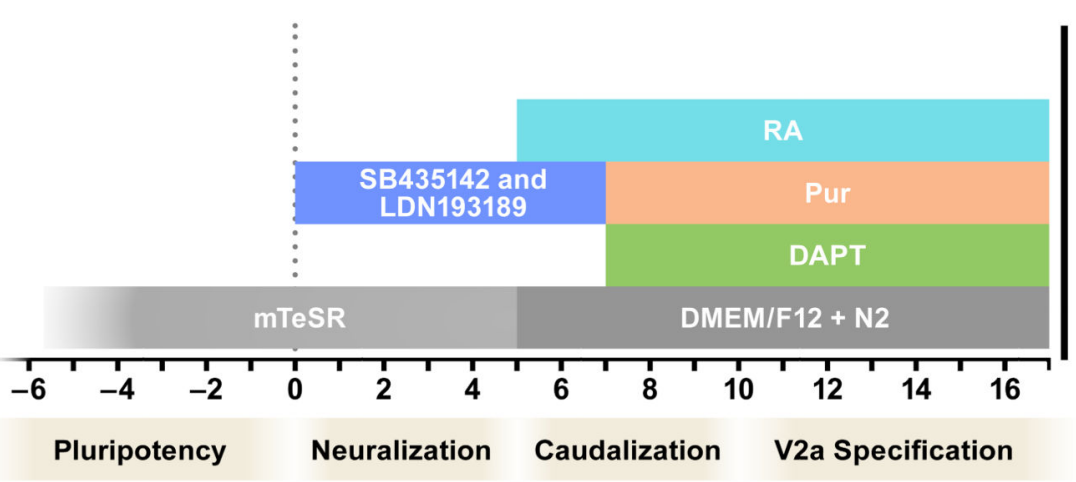

Select: 3A

Reaggregate: Box 1

Mature: 4A

Characterization: Step 5

Flow Cytometry: 5A

Immunocytochemistry: $5 B$

qPCR: 5C

Replate: 3B

Freeze/Thaw: Box 2

Mature: 4B

C

\begin{tabular}{|c|c|c|c|c|c|}
\hline $\begin{array}{l}\text { Differentiation } \\
\text { Stage }\end{array}$ & Species & Day(s) & Base Media & Supplements & $\begin{array}{l}\text { Procedure } \\
\text { Number }\end{array}$ \\
\hline \multirow{2}{*}{ Pluripotency } & Mouse & pre d0 & Complete & Lif & $1 \mathrm{~A}$ \\
\hline & Human & pre d0 & $\mathrm{mTeSR}$ & Rocki with passage & 1B \\
\hline \multirow{2}{*}{ Neuralization } & Mouse & D0-D2 & DFK5 & $10 \mathrm{nM} R A+1 \mu \mathrm{M}$ Pur & 2Ai-iii \\
\hline & Human & D0-D5 & mTeSR & SB + LDN & $2 \mathrm{Bi}-\mathrm{v}$ \\
\hline \multirow{3}{*}{ Caudalization } & Mouse & D2-D4 & DFK5 & $10 \mathrm{nM} R A+1 \mu \mathrm{M}$ Pur & 2Aiv-vii \\
\hline & \multirow{2}{*}{ Human } & D5-D7 & \multirow{2}{*}{$\mathrm{NIM}+\mathrm{AA}+\mathrm{BDNF}$} & $\mathrm{SB}+\mathrm{LDN}+100 \mathrm{nM} \mathrm{RA}$ & 2Bvi \\
\hline & & D7-D10 & & $100 \mathrm{nM}$ RA + $100 \mathrm{nM}$ Pur+ $1 \mu \mathrm{M}$ DAPT & 2Bvii \\
\hline \multirow{2}{*}{ V2a Specification } & Mouse & D4-D6 & DFK5 & $10 \mathrm{nM}$ RA + $1 \mu \mathrm{M}$ Pur+ $5 \mu \mathrm{M}$ DAPT & 2Aviii-xi \\
\hline & Human & D10-D17 & $\mathrm{NIM}+\mathrm{AA}+\mathrm{BDNF}$ & 100 nM RA + 100 nM Pur+ $1 \mu$ M DAPT & 2Bvii-viii \\
\hline
\end{tabular}

Figure 2 |.

Schematic of mouse and human V2a interneuron differentiation protocol. (a) Timeline for V2a interneuron differentiation from mouse ESCs. At the end of the differentiation, the cultures can be selected, reaggregated with $\mathrm{OLIG}^{+}$cells, and/or matured. (b) Timeline for V2a interneuron differentiation from human PSCs. At the end of the differentiation, the cultures can be replated, cryopreserved, and/or matured. Both the mouse and human differentiations can be characterized using flow cytometry, immunocytochemistry, and/or qPCR. (c) Table describing differentiation stages, media compositions, and corresponding procedure number for the mouse and human protocols. 
a
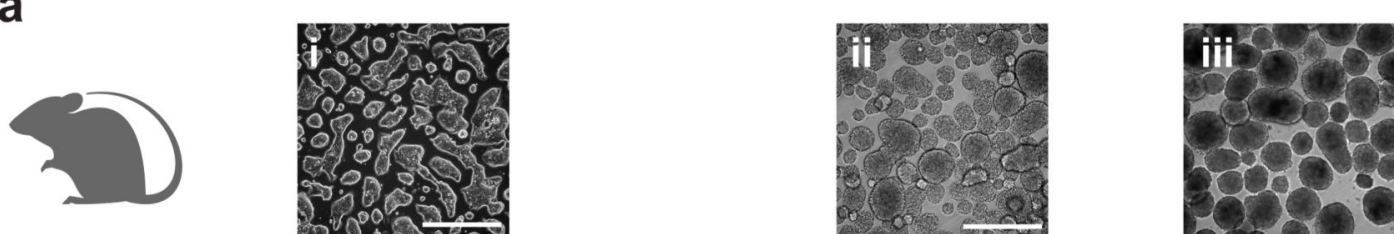

mESC

D2

D4

D6

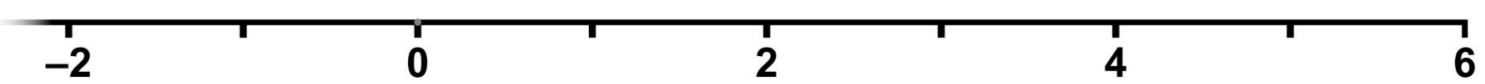

6

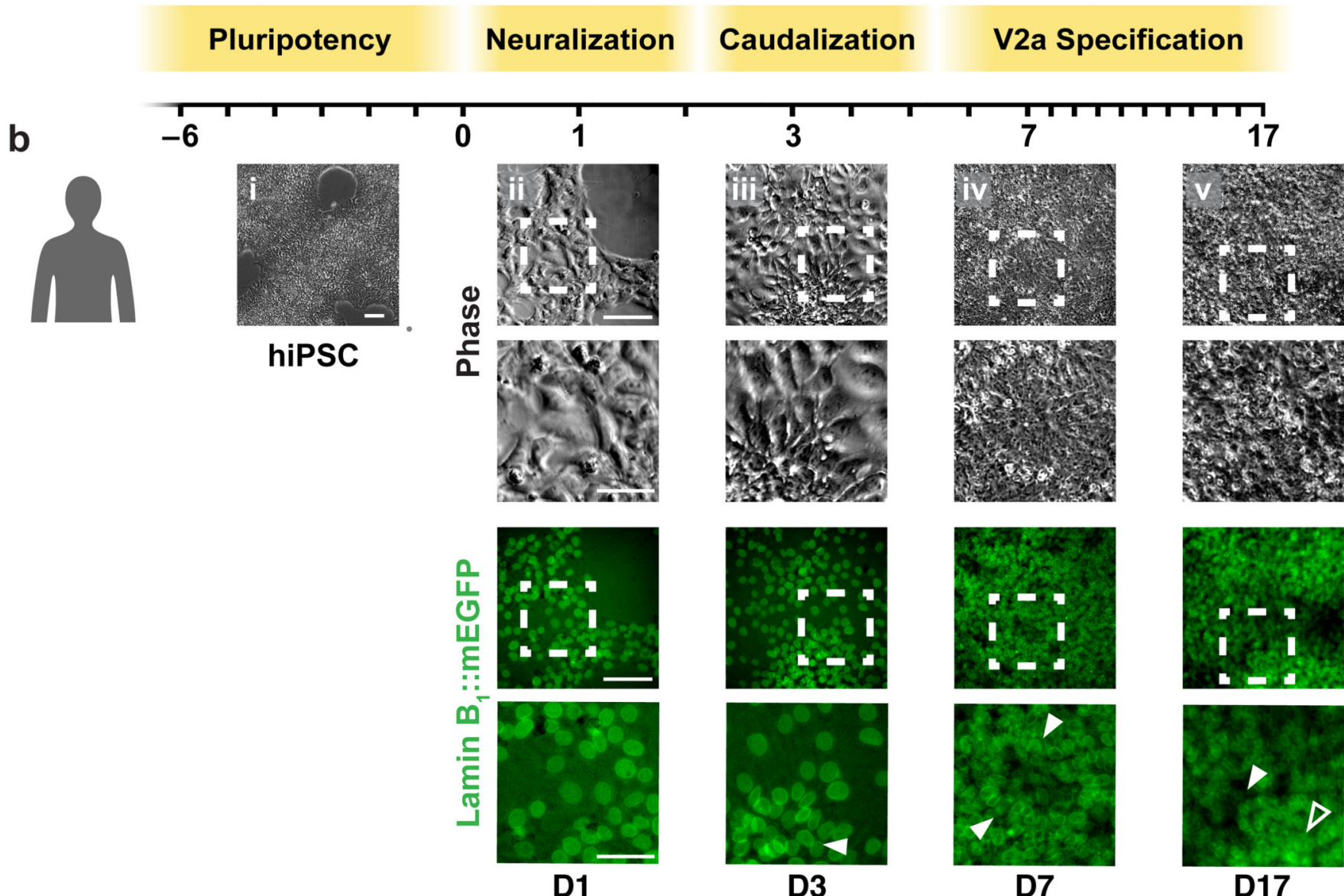

Figure 3 |.

Phase and fluorescent imaging throughout the mouse and human differentiations. ( $\left.\mathbf{a}_{\mathbf{i} \text {-iv }}\right)$ (i) Phase images of mouse ESCs. Scale bar $=500 \mu \mathrm{m}$. (ii-iv) Phase images of aggregates throughout the differentiation on days 2 (ii), 4 (iii), and 6 (iv). Scale bar $=500 \mu \mathrm{m}$. ( $\mathbf{b}_{\mathbf{i - v}}$ ) (i) Phase images of Lamin-B $1:$ mEGFP human PSCs (i). Scale bar $=100 \mu \mathrm{m}$. (ii-iv) Phase and fluorescent throughout the differentiation on days 1(ii), 3 (iii), 7 (iv), and 17. Scale bar $=100$ $\mu \mathrm{m},(\mathbf{v})$. Dotted lines demarcate areas that are enlarged below. Scale bar $=50 \mu \mathrm{m}$. ( $\left.\mathbf{b}_{\mathrm{iii}}\right)$ Arrowheads demarcate nuclei that have begun to condense. ( $\mathbf{b}_{\mathbf{i v}}$ ) Arrowheads demarcate elongated and radially aligned nuclei. $\left(\mathbf{b}_{\mathbf{v}}\right)$ Solid arrowheads demarcate valleys in the culture and empty arrowheads demarcate peaks in the culture. 

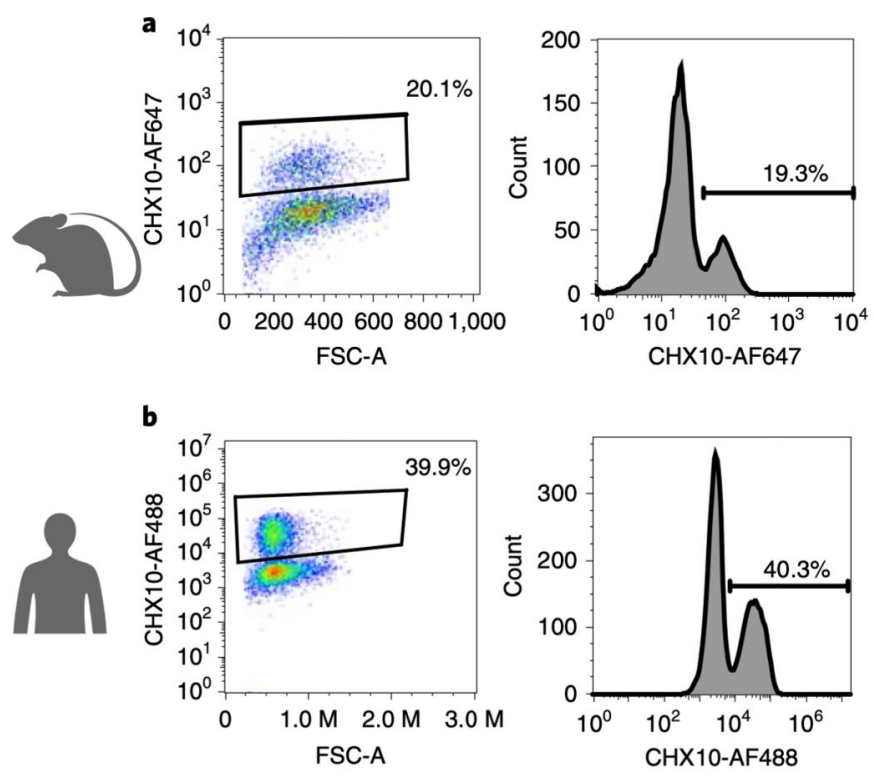
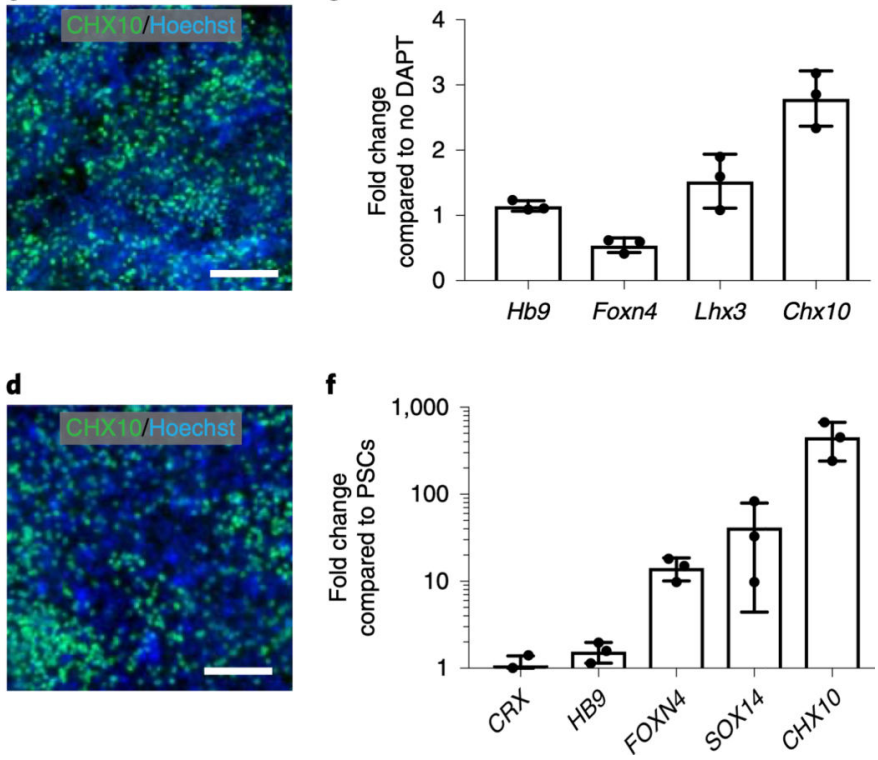

Figure $4 \mid$.

End point analyses of mouse and human differentiations. (a) Flow cytometry analysis of CHX10 at D6 of mouse differentiation represented in a scatter plot and histogram. (b) Flow cytometry analysis of CHX10 at D17 of human differentiation represented in a scatter plot and histogram. (c) Immunocytochemistry for CHX10 (green) and Hoechst (blue) on plated mouse aggregates at D6. Scale bar $=100 \mu \mathrm{m}$, (d) Immunocytochemistry for CHX10 (green) and Hoeschst (blue) on human monolayer cultures at D17. Scale bar $=100 \mu \mathrm{m}$, (e) qPCR on D6 mouse cultures. Reported fold change is compared to cultures that did not receive DAPT, $\mathrm{n}=3$ from distinct samples. (f) qPCR on D17 human cultures. Reported fold change is compared to PSCs. Error bars indicate standard deviation, $n=3$ from distinct samples. 

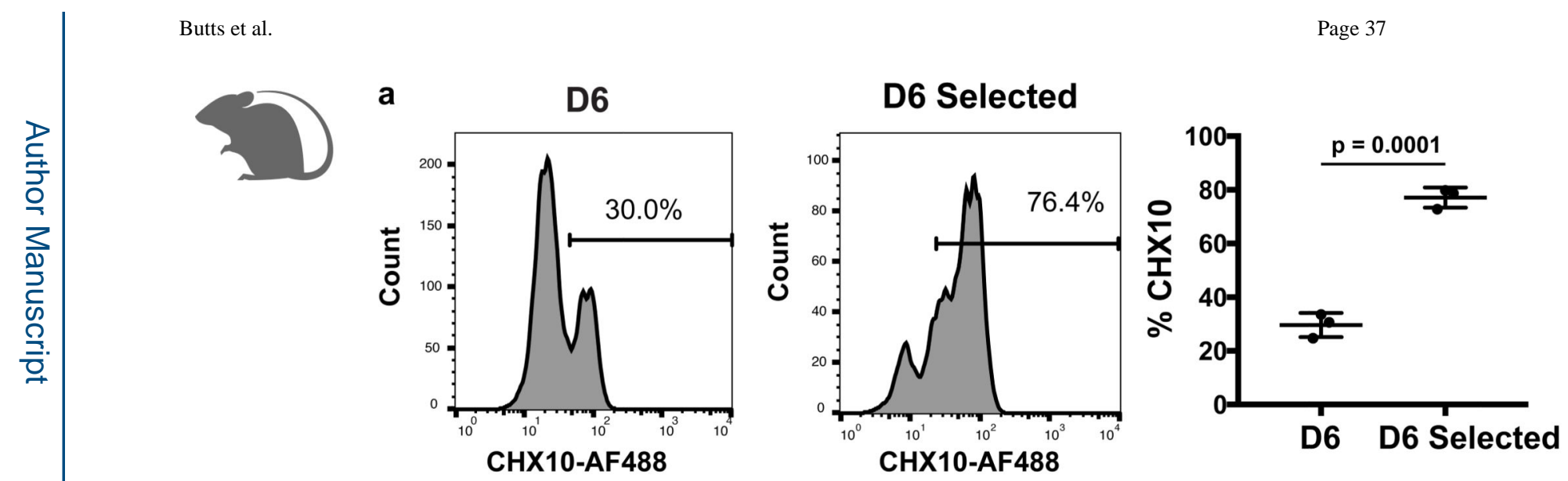

b

D20
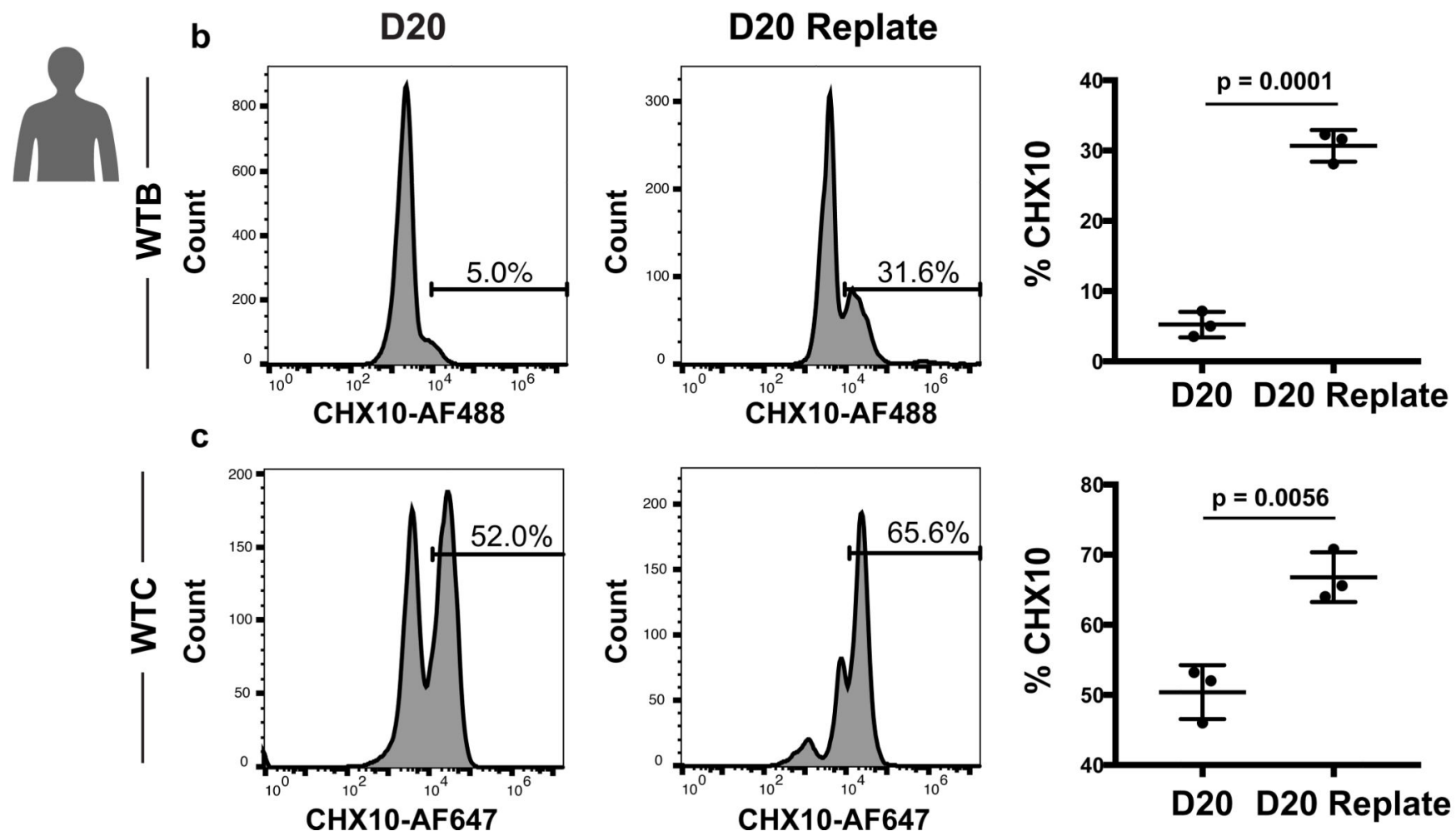

Figure 5 |.

Enrichment of V2a interneuron populations. (a) Flow cytometry analysis of CHX10 in D6 unselected and selected mouse cultures. Data from Iyer et al. ${ }^{9}$ Flow cytometry analysis of CHX10 in D20 cultures that have or have not been replated using WTB hiPSCs (b) or WTC hiPSCs (c). P-value determined by unpaired two-tailed t test. Error bars indicate standard deviation from mean, $\mathrm{n}=3$ from distinct samples. 


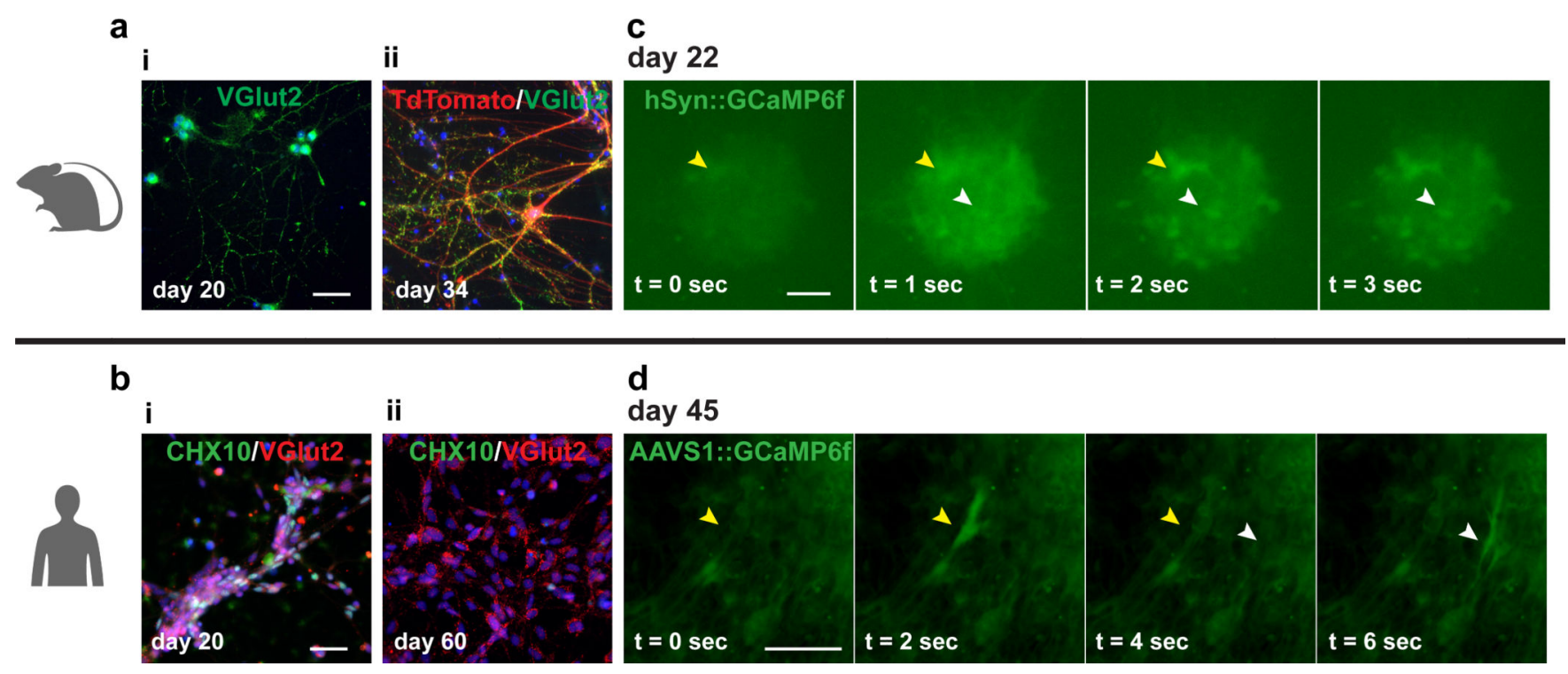

Figure 6 .

Maturation of V2a interneuron cultures. ( $\left.\mathbf{a}_{\mathbf{i}}\right)$ Immunocytochemistry of selected mouse V2a interneurons at D18 stained for vesicular glutamate transporter 2 (VGlut2, green) and

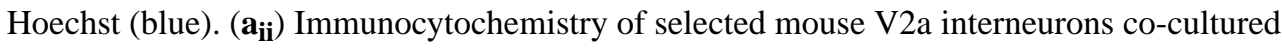
with Olig2 $2^{+}$cells at D34 stained for VGlut2 (green), Hoechst (blue), and imaged for TdTomato (red) to identify V2a interneurons. ( $\left.\mathbf{b}_{\mathbf{i}}\right)$ Immunocytochemistry of V2a interneurons at D20 stained for CHX10 (green), VGlut2 (red), and Hoechst (blue). ( $\left.\mathbf{b}_{\text {ii }}\right)$ Immunocytochemistry of V2a interneurons at D60 stained for CHX10 (green), VGlut2 (red), and Hoechst (blue). (c) Fluorescent imaging of hSyn-GCaMP6f in mouse V2a-Olig2 aggregates at D22. Arrowheads demarcate areas of calcium flux. (d) The differentiation was performed with the WTC hiPSC cell line harboring the genetically-encoded calcium sensor GCaMP6f. Fluorescent imaging of constitutive GCaMP6f in human V2a interneuron cultures at D45. Arrowheads demarcate areas of calcium flux. All scale bars $=50 \mu \mathrm{m}$. 

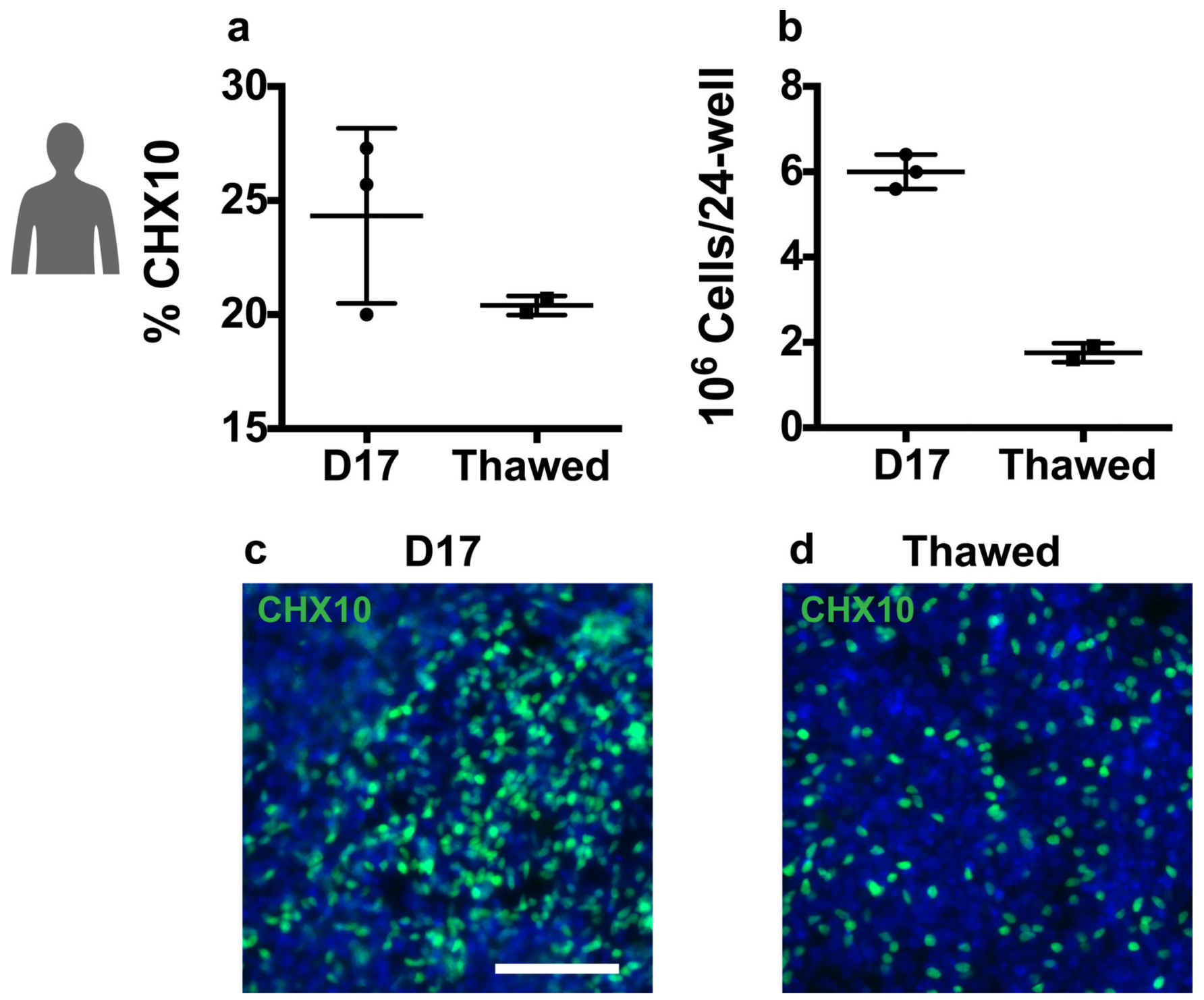

Figure $7 \mid$.

Cryopreservation of human V2a interneuron cultures. (a) Flow cytometry analysis of CHX10 at D17 and after three days of recovery following cryopreservation. (b) Total viable cell count at D17 and after three days of recovery following cryopreservation. Error bars indicate standard deviation from mean, $\mathrm{n}=3$ for $\mathrm{D} 17$ and $\mathrm{n}=2$ for thawed from distinct samples. Immunocytochemistry of CHX10 (green) and Hoechst (blue) at D17 (c) and three days of recovery following cryopreservation (d). Scale bar $=50 \mu \mathrm{m}$. 
a
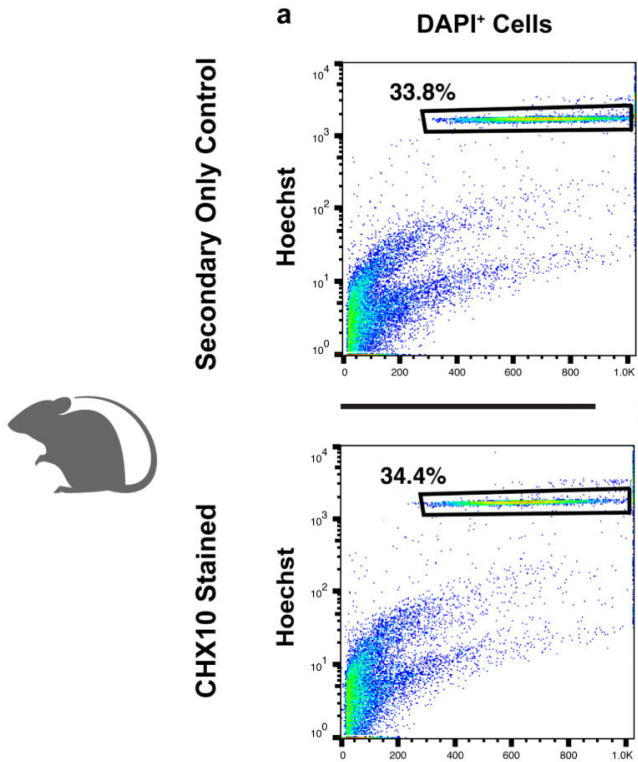

b

CHX10+ Cells

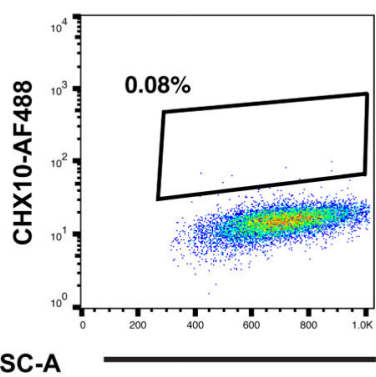

FSC-A

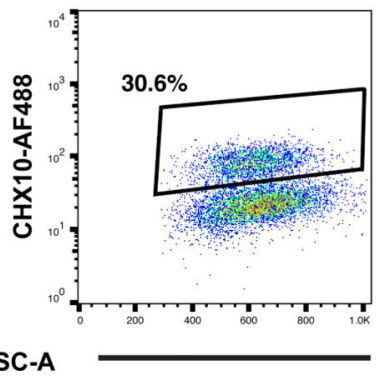

c

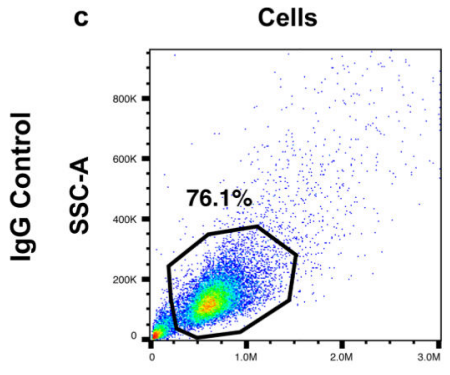

.

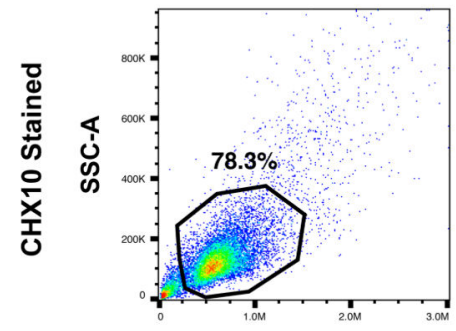

d
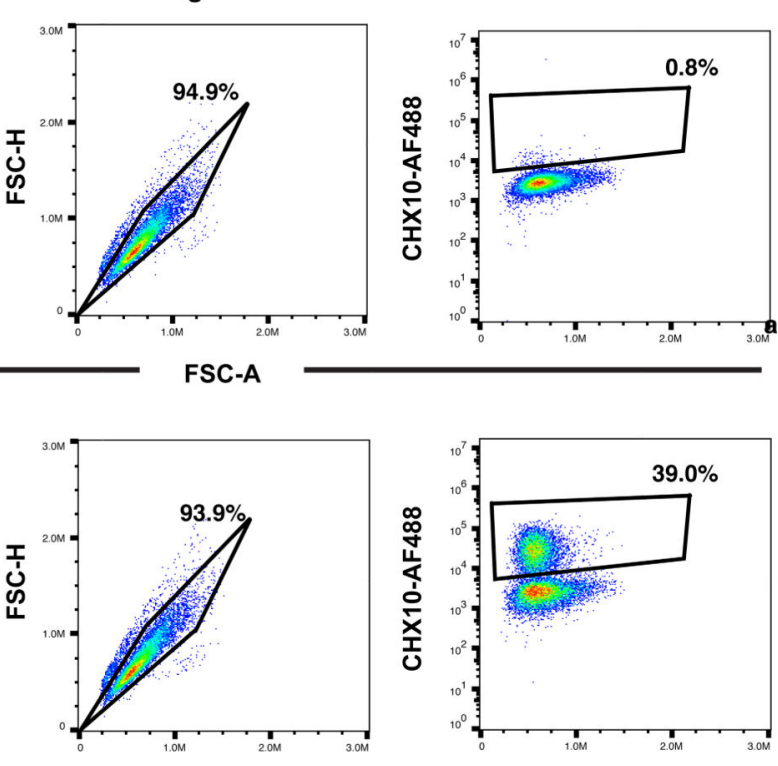

Figure 8 |.

Flow cytometry gating strategy. (a) Gating of Hoechst ${ }^{+}$cells in the secondary only control and the CHX10 stained mouse cells. (b) Gating of $\mathrm{CHX}^{+} 0^{+}$cells in the secondary only control and the CHX10 stained mouse cells. (c) Gating of cell population using forward scattered area (FSC-A) vs. side scattered area (SSC-A) in the IgG control and the CHX10 stained human cells. (d) Gating of single cells using FSC-A vs. forward scattered height (FSC-H) in the IgG control and the CHX10 stained human cells. (e) Gating of the CHX10 cells in the IgG control and the CHX10 stained human cells. 

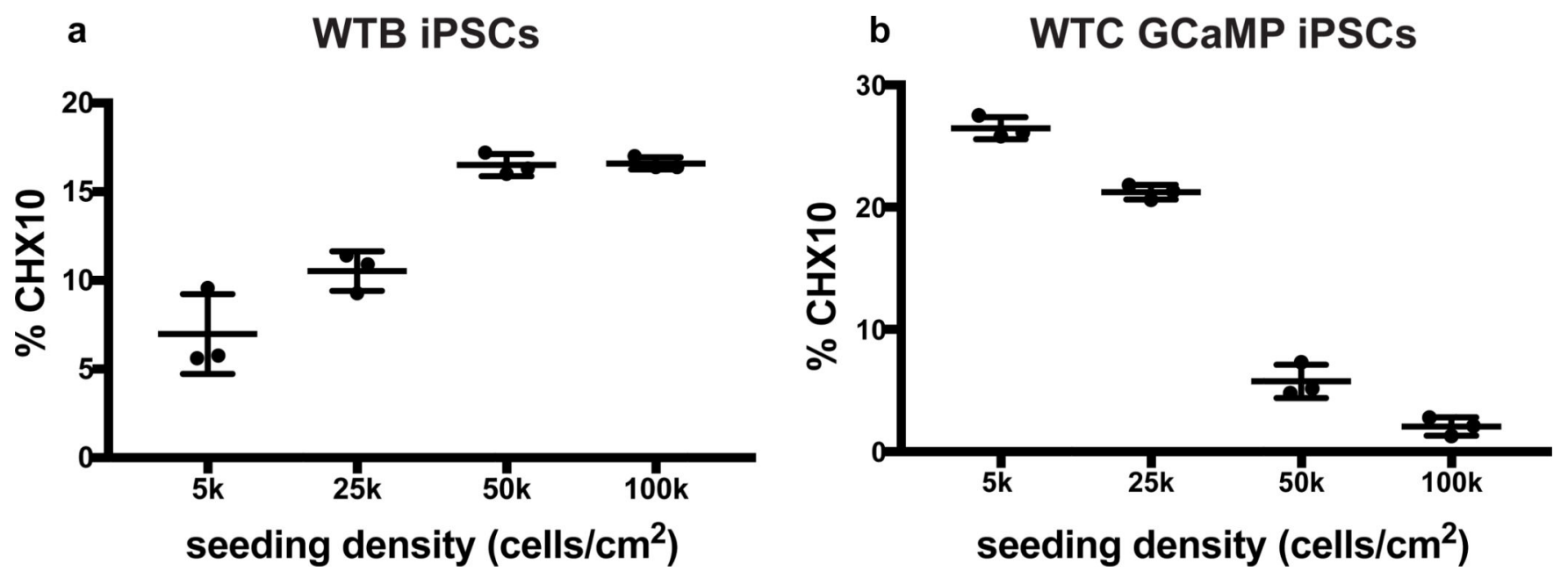

Figure 9 |.

Seeding density is critical to human V2a interneuron differentiation. Flow cytometry analysis of CHX10 at D17 using varying initial seeding densities with WTB iPSCs (a) and WTC GCaMP iPSCs (b). Error bars indicate standard deviation from mean, $\mathrm{n}=3$ from distinct samples. 

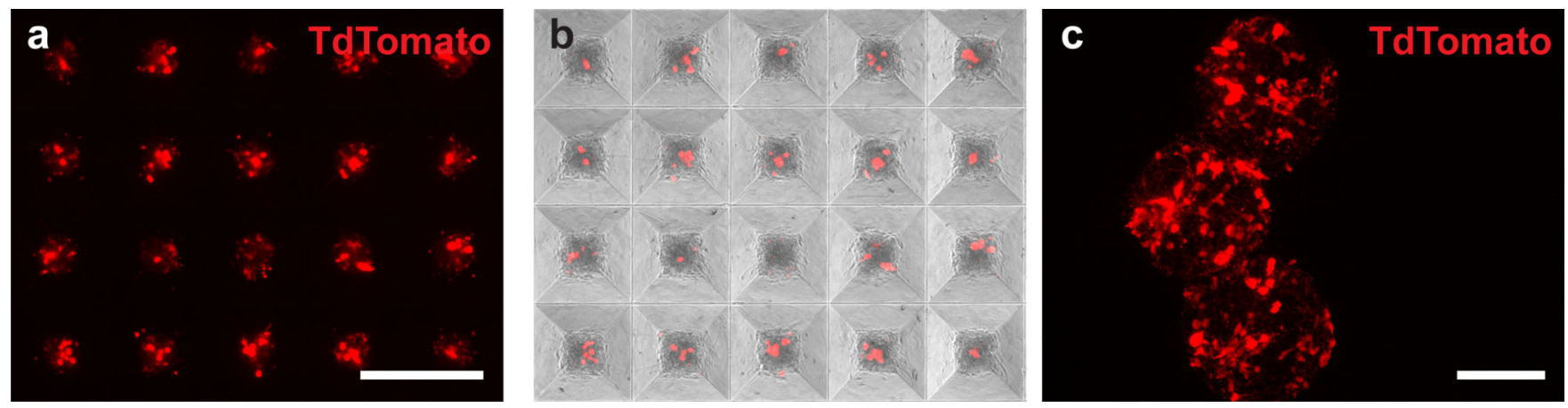

Figure $10 \mid$.

Mouse V2a interneuron aggregate formation. Fluorescent imaging of aggregates formed from selected V2a interneurons (TdTomato, red) $(\mathbf{a})$ with Olig2 $^{+}$cells (phase) $(\mathbf{b})$ scale bar $=$ $400 \mu \mathrm{m}$. (c) Fluorescent imaging of plated V2a-Olig2 aggregates. Scale bar $=100 \mu \mathrm{m}$ 
Table 1|

Antibodies used for immunocytochemistry and flow cytometry.

\begin{tabular}{|l|l|l|l|l|}
\hline Primary Antibodies \\
\hline Target & Cat. Number & Species & Dilution & Vendor \\
\hline Chx10 & sc-374151 & Mouse & $1: 1000$ & Santa Cruz Biotechnology \\
\hline$\beta$-III-Tubulin & MRB-435P & Rabbit & $1: 1000$ & BioLegend \\
\hline VGlut 2 & 135403 & Rabbit & $1: 500$ & Synaptic Systems \\
\hline \multicolumn{5}{|l|}{} \\
\hline Secondary Antibodies & Cat. Number & Dilution & Vendor \\
\hline Target & A-31572 & $1: 200$ & Invitrogen \\
\hline Alexa 488 donkey anti mouse IgG & A-21202 & $1: 200$ & Invitrogen \\
\hline Alexa 555 donkey anti Rabbit IgG & A- $1: 200$ & Invitrogen \\
\hline Alexa 647 donkey anti mouse IgG & A-31571 & $1: 0$ & \\
\hline \multicolumn{5}{|l|}{} \\
\hline Isotype Controls & & Cat. Number & Dilution & Vendor \\
\hline Target & AB002 & N/A & R\&D \\
\hline Mouse IgG1 Isotype Control
\end{tabular}


Table 2|

Primers used in qPCR for mouse and human analyses.

\begin{tabular}{|l|c|c|c|}
\hline Primer & Mouse (Assay ID) & \multicolumn{2}{|c|}{ Human (Sequence) } \\
\hline CHX10 & Mm00432549_m1 & F: CGGCGACACAGGACAATCTT & R: CCTGTATCCTGTCTTCCGGC \\
\hline CRX & N/A & F: CCTTCTGACAGCTCGGTGTT & R: TGGTGTACTTCAGCGGTCAC \\
\hline FOXN4 & Mm00521694_m1 & F: CGTACAGCTGTCTGATCGCC & R: GGAGCCGCTCATCTTGTTCT \\
\hline$H B 9$ & Mm01222622_m1 & F: TCTCTTAACGGGAAGGGGCA & R: CTAATTCAGGGCGCTCTCGG \\
\hline LHX3 & Mm01330619_g1 & N/A & N/A \\
\hline SOX14 & N/A & F: GAACCCTTGCACTCCCTACC & R: TCGATGTATGGCCGCTTCTC \\
\hline
\end{tabular}


Table 3:

Troubleshooting

\begin{tabular}{|c|c|c|c|}
\hline Step & Problem & Possible Reason & Solution \\
\hline 1A(ii) & $\begin{array}{l}\text { mESCs are not reaching } \\
\text { confluency at the predicted } \\
\text { time }\end{array}$ & $\begin{array}{l}\text { - Need to seed a different ratio } \\
\text { at passage }\end{array}$ & $\begin{array}{l}\text { - The passage ratio can be changed in order to increase cell } \\
\text { density or shift when passaging needs to be conducted. Passage } \\
\text { at a } 1: 3 \text { ratio to achieve confluence after } 1 \text { day. Passage at a 1:8 } \\
\text { ratio to achieve confluence after } 3 \text { days. }\end{array}$ \\
\hline 1B(ii) & $\begin{array}{l}\text { hPSCs not reaching } \\
\text { confluency }\end{array}$ & $\begin{array}{l}\text { - Initial seeding density is too } \\
\text { low }\end{array}$ & $\begin{array}{l}\text { - Try a few different seeding densities when working with a new } \\
\text { cell line. }\end{array}$ \\
\hline 2B(vii) & $\begin{array}{l}\text { Cell layers are detaching } \\
\text { from plate during human } \\
\text { differentiation }\end{array}$ & $\begin{array}{l}\text { - Media is too cold } \\
\text { - Re-feeding is dislodging the } \\
\text { layers } \\
\text { - Incompatible Matrigel lot } \\
\text { - Inefficient Matrigel coating }\end{array}$ & $\begin{array}{l}\text { - Make sure media is at least at room temperature before } \\
\text { feeding. } \\
\text { - Make sure the pipette dispenser is set to the slowest setting } \\
\text { before feeding. } \\
\text { - Test the differentiation with different Matrigel lots. } \\
\text { - Use fresh Matrigel and be sure to coat for } 24 \text { hours. }\end{array}$ \\
\hline $3 \mathrm{~A}(\mathrm{i})$ & $\begin{array}{l}\text { Not enough V2a } \\
\text { interneurons after selection } \\
\text { for subsequent experiments }\end{array}$ & $\begin{array}{l}\text { - Need to induce more } \mathrm{V} 2 \mathrm{a} \\
\text { interneurons }\end{array}$ & $\begin{array}{l}\text { - To obtain the necessary high seeding density, multiple } \\
\text { differentiation plates may need to be pooled together depending } \\
\text { on the surface for replating or aggregation. }\end{array}$ \\
\hline 4B(iii) & $\begin{array}{l}\text { Cell layers ball up during } \\
\text { maturation }\end{array}$ & $\begin{array}{l}\text { The cells have been on the same } \\
\text { plate for too long and may be } \\
\text { losing the matrix keeping the } \\
\text { cells attached to the plate }\end{array}$ & $\begin{array}{l}\text {-Dissociate and replate the cultures onto fresh well plates. Note } \\
\text { that this will dilute out the } \mathrm{CHX} 10^{+} \text {cells. }\end{array}$ \\
\hline $5 \mathrm{~A}(\mathrm{ix})$ & $\begin{array}{l}\text { Low CHX10 percentage in } \\
\text { human differentiations }\end{array}$ & $\begin{array}{l}\text { - Initial seeding density is } \\
\text { inappropriate } \\
\text { - Incompatible Matrigel lot }\end{array}$ & $\begin{array}{l}\text { - May need to test different lots of Matrigel to see what supports } \\
\text { a neuronal differentiation. } \\
\text { - Test a range of seeding densities for each cell line. }\end{array}$ \\
\hline $5 \mathrm{~B}(\mathrm{i})$ & $\begin{array}{l}\text { Cell layer peeling during } \\
\text { staining }\end{array}$ & $\begin{array}{l}\text { Cell layers are releasing during } \\
\text { fixation and wash steps }\end{array}$ & $\begin{array}{l}\text { Leave } 500 \mu \mathrm{L} \text { of media in the wells and add } 500 \mu \mathrm{L} \text { of } 4 \% \text { PFA } \\
\text { into the wells to fix. Fix for } 1 \text { hour instead of } 30 \text { minutes. } \\
\text { During wash steps, a transfer pipette may be used to carefully } \\
\text { exchange liquids. }\end{array}$ \\
\hline $5 B($ vii) & $\begin{array}{l}\text { Speckled signal in } \\
\text { immunostaining }\end{array}$ & $\begin{array}{l}\text { Excess secondary antibodies } \\
\text { aggregate and do not wash out }\end{array}$ & $\begin{array}{l}\text { Filter the solution with the secondary antibody to remove any } \\
\text { aggregates. If the target has low abundance, try a lower } \\
\text { concentration of secondary antibody }(<1: 200) \text {. }\end{array}$ \\
\hline Box 2: 6 & $\begin{array}{l}\text { Low cell survival post- } \\
\text { thaw }\end{array}$ & Cell death during thaw & Do not let the vial of cells completely thaw in the water bath. \\
\hline Box 2: 8 & $\begin{array}{l}\text { Low cell survival post- } \\
\text { thaw }\end{array}$ & Excess cell debris after thaw & $\begin{array}{l}24 \text { hours after thaw carefully aspirate the media and wash with } \\
0.5 \mathrm{ml} \text { PBS. Aspirate PBS and add fresh NIM. }\end{array}$ \\
\hline
\end{tabular}

